\title{
Effects of Smooth Divergence-Free Flows on Tracer Gradients and Spectra: Eulerian Prognosis Description
}

\author{
VALENTIN Resseguier, ${ }^{\mathrm{a}}$ Bertrand CHAPRON, ${ }^{\mathrm{b}}$ AND ETIENNE MÉmin ${ }^{\mathrm{c}}$ \\ ${ }^{\text {a }}$ Lab, SCALIAN, Rennes, France \\ ${ }^{\mathrm{b}}$ LOPS, Ifremer, Plouzané, France \\ ${ }^{\mathrm{c}}$ Fluminance Team, Inria, Rennes, France
}

(Manuscript received 20 January 2021, in final form 19 October 2021)

\begin{abstract}
Ocean eddies play an important role in the transport of heat, salt, nutrients, or pollutants. During a finitetime advection, the gradients of these tracers can increase or decrease, depending on a growth rate and the angle between flow gradients and initial tracer gradients. The growth rate is directly related to finite-time Lyapunov exponents. Numerous studies on mixing and/or tracer downscaling methods rely on satellite altimeter-derived ocean velocities. Filtering most oceanic small-scale eddies, the resulting smooth Eulerian velocities are often stationary during the characteristic time of tracer gradient growth. While smooth, these velocity fields are still locally misaligned, and thus uncorrelated, to many coarse-scale tracer observations amendable to downscaling [e.g., sea surface temperature (SST), sea surface salinity (SSS)]. Using finite-time advections, the averaged squared norm of tracer gradients can then only increase, with local growth rate independent of the initial coarse-scale tracer distribution. The key mixing processes are then only governed by locally uniform shears and foldings around stationary convective cells. To predict the tracer deformations and the evolution of their second-order statistics, an efficient proxy is proposed. Applied to a single velocity snapshot, this proxy extends the Okubo-Weiss criterion. For the Lagrangian-advection-based downscaling methods, it further successfully predicts the evolution of tracer spectral energy density after a finite time, and the optimal time to stop the downscaling operation. A practical estimation can then be proposed to define an effective parameterization of the horizontal eddy diffusivity.

SIGNIFICANCE STATEMENT: An analytical formalism is adopted to derive new exact and approximate relations that express the clustering of tracers transported by upper-ocean flows. This formalism bridges previous Eulerian and Lagrangian approaches. Accordingly, for slow and smooth upper-ocean flows, a rapid prognosis estimate can solely be performed using single-time velocity field observations. Well suited to satellite-altimeter measurements, it will help rapidly identify and monitor mixing regions occurring in the vicinity of ocean eddy boundaries.
\end{abstract}

KEYWORDS: Mixing; Vortices; Diagnostics

\section{Introduction}

Since the first images from space, the attention of both theoreticians and remote sensing scientists has been triggered by the abundance of various ocean tracer patterns and signatures in the mesoscale and submesoscale $(1-50 \mathrm{~km}$ ) ranges (e.g., Gower et al. 1980; Lesieur and Sardouny 1981). From precise satellite measurements of the ocean topography and its related dynamics, coherent eddies have since been identified to stretch and fold tracers, leading to the generation of often very spectacular upper-ocean intricate tracer distributions. Nowadays, combined satellite altimeter measurements satisfactorily detail the large-scale ocean dynamics (Klein et al. 2019). But the ocean's mesoscale (10-100 km) and submesoscale $(<10 \mathrm{~km})$ variability and energy are still challenging to map with conventional radar altimeters. Indeed, the narrow illuminated swath of each instrument precludes precise mapping, regardless of the orbital configuration (Dufau et al. 2016). To date, global direct quantification of horizontal dispersion and mixing at such scales is thus not available.

Corresponding author: Valentin Resseguier, valentin.resseguier@ scalian.com
Nonetheless, a now-common strategy is to derive smallscale tracer structures and so-called Lagrangian coherent structures from the available smooth altimeter-derived velocities (e.g., Price et al. 2006; Lehahn et al. 2007). Indeed, using a Lagrangian dynamical framework, an initial larger-scale tracer field can be advected on higher-resolution grids, generating much smaller-scale patterns (Aref 1984; Pierrehumbert and Yang 1993). Typical moderate- to large-scale ocean cyclonic and anticyclonic eddies trap and advect fluid parcels over weeks to months. As pictured, with time, these fluid parcels with different origins, temperature, and salinity, and possibly different biogeochemical properties and/or contaminant loadings, come closer to sharpening fronts but also to possibly diluting their properties, and promote transformative chemical reactions. Stirring effect first characterizes the development of elongated structures, illustrated by Welander (1955, see his Fig. 2), using a simple velocity field to produce spectacular distortions. Initial patches, small compared to the length scale of the deforming flow field, become subject to translation, rotation, and shearing. With time, deformation is significant. Increasingly long and thin filaments wrap around the eddy and possibly fold. Folds appear where the velocity gradient is perpendicular to the stream direction. Accordingly, at a given scale of observation, mixing can be associated with 
processes that act to minimize filament thinning and dilute sharp differences (gradients). The resulting deformation of tracer isolines is thus associated with strengthening or weakening of small-scale structures also captured in the high-wavenumber part of the tracer spectra.

In this paper, the motivation is first to present an analytical framework to derive exact and approximate results for the evolution of tracer gradients after a finite-time advection. After a long-time advection by a smooth and slowly varying incompressible flow, the expected growth of passive tracer gradients can indeed be theoretically obtained, and subsequently the related evolution of tracer high-wavenumber spectra. Analytically, local and global stretching and folding properties can then be diagnosed without time integration. From a practical point of view, only a single snapshot of a velocity field is required.

This result provides a convenient diagnosis that fully applies to estimated smooth velocities from altimeter-derived sea surface height (SSH) measurements. The Eulerian prognosis description then explains how an initial tracer field, sea surface temperature (SST) or sea surface salinity (SSS), must be low-pass filtered in forward-backward Lagrangian advection operations (Rogé et al. 2015). Accordingly, the time of advection and the low-pass-filter bandwidth are directly linked. Following this development, an exact relation can also be determined to provide more rigorous constrains to the heuristic choices used in Dencausse et al. (2014) and Rogé et al. (2015). This can be compared to estimates inferred from the knowledge of the Rossby deformation radius or the mean squared vorticity (Berti and Lapeyre 2014).

In section 2, we recall and propose exact theoretical results to study tracer mixing. Section 3 focuses in our case study: downscaling and mixing analyses with coarse-scale tracers and surface current observations. Associated stretching and folding diagnostics are derived, and folding and shearing time defined. Besides, the proposed analysis also conveniently provides further understanding in identifying regions with motions either dominated by rotation or by stretching where two points become closer or diverge. Mixing can then occur when folding is associated with stretching effects to strongly strengthen tracer gradients. In section 4 , the evolution of the tracer high-wavenumber spectral tail is presented. The norm of the averaged tracer gradient is shown to control the evolution of the tracer spectral tail. Eulerian descriptors are then proposed to monitor the aforementioned Lagrangian advection downscaling methodology. Based on these proposed developments, a practical estimation of the horizontal diffusivity is derived to help constrain subgrid parameterizations of large-scale flow simulations. In section 5, numerical experiments are used to illustrate these analytical developments. Analyses are finally performed using altimeter-derived smooth ocean velocities. Conclusions follow in section 5 .

\section{Exact mixing properties}

Hereafter, exact results are derived to describe the evolution of the average of the gradient squared norm of an advected tracer $T$ :

$$
\overline{\|\nabla T\|^{2}}
$$

where the averaging operator $\bullet$ is defined for every function $q$ as $\bar{q}=(1 / S) \int_{\Omega} q$ with integration over the two-dimensional spatial domain $\Omega$ of finite area $S$.

\section{a. Stretching and the Cauchy-Green tensor}

Given a two-dimensional velocity field $\mathbf{v}$, the flow $\boldsymbol{\phi}$-also called Lagrangian displacement - is defined as

$$
\boldsymbol{\phi}\left(\mathbf{x}_{0}\right)=\boldsymbol{\phi}\left(\mathbf{x}_{0}, t\right)=\mathbf{x}_{0}+\int_{0}^{t} d t^{\prime} \mathbf{v}\left[\boldsymbol{\phi}\left(\mathbf{x}_{0}, t^{\prime}\right), t^{\prime}\right]
$$

For a divergence-free velocity, $\nabla \cdot \mathbf{v}=0$, we have $\operatorname{det}\left(\nabla \boldsymbol{\phi}^{\mathrm{T}}\right)=1$, where $\left(\nabla \boldsymbol{\phi}^{\mathrm{T}}\right)$ is the spatial gradient tensor of the flow. Subsequently, the right Cauchy-Green deformation tensor, $\nabla \boldsymbol{\phi}^{\mathrm{T}}\left(\nabla \boldsymbol{\phi}^{\mathrm{T}}\right)^{\mathrm{T}}$, and its inverse shall have two real and identical strictly positive eigenvalues. Only the stable direction, corresponding to the eigenvector associated with the eigenvalue smaller than 1 , and the unstable direction, corresponding to the eigenvector associated with the eigenvalue larger than 1 , are switched. Along the stable (resp. unstable) direction, the distance between two points decreases (resp. increases). More details of this classical analysis of the Cauchy-Green tensor are recalled in appendix $\mathrm{A}$.

In appendix B, we derived the new following compact expression of the transported tracer gradient $\nabla T$ as a function of the initial gradient field $\nabla T_{0}$ and the flow $\boldsymbol{\phi}$ :

$$
\overline{\|\nabla T\|^{2}}-\overline{\left\|\nabla T_{0}\right\|^{2}}=\overline{\left\|\nabla T_{0}\right\|^{2} \alpha^{2}\left[1+\frac{\beta}{\alpha} \cos \left(2 \theta_{T_{0}}^{\phi}\right)\right]},
$$

where

$$
\alpha^{2}=\frac{1}{2}\left\|\nabla \boldsymbol{\phi}^{\mathrm{T}}\right\|^{2}-1 \geq 0, \beta^{2}=\alpha^{2}+2
$$

using the Frobenius matrix norm, and $\theta_{T_{0}}^{\boldsymbol{\phi}}$ stands for the angle between the tracer gradient and the compressive (stable) direction of the direct flow. The Cauchy-Green tensor and the initial tracer gradient completely determine the averaged squared norm of advected tracer gradients. The advection acts to globally increase (decrease) the tracer gradient norm if the initial tracer gradient is locally close enough to the stable (unstable) direction of the direct flow. This corresponds to $\theta_{T_{0}}^{\boldsymbol{\phi}}$ close to 0 modulo $\pi$ or $\pi / 2$ modulo $\pi$, respectively. This is modulated by the initial amplitude of the tracer gradients, a growth rate $\alpha^{2}$ and a factor $(\beta / \alpha)=\sqrt{1+\left(2 / \alpha^{2}\right)} \geq 1$. Note, $\alpha$ and $\beta$ do not explicitly depend on the tracer.

The largest finite time Lyapunov exponent (FTLE) (Haller and Yuan 2000; Thiffeault and Boozer 2001; Haller 2005; Haller and Sapsis 2011) is $\Lambda=(1 / 2 t) \log \left\{1+\alpha^{2}[1+(\beta / \alpha)]\right\}$. In particular, when both the largest FTLE $\Lambda$ and the time $t$ are large, the term $\alpha^{2}$ is large and $(\beta / \alpha)=\sqrt{1+\left(2 / \alpha^{2}\right)}$ is small, leading to the approximation $\Lambda \approx(1 / t) \log (\alpha)$. Therefore, the FTLE ridges - often considered as proxies of mixing barriers - coincide with the $\alpha$ ridges. 


\section{b. Stretching expressed with mesochronic velocity}

Mezić et al. (2010) introduce a mesochronic velocity defined as the velocity, time-averaged along a trajectory:

$$
\breve{\mathbf{v}}\left(\mathbf{x}_{0}, t\right)=\left[\begin{array}{c}
\breve{u}\left(\mathbf{x}_{0}, t\right) \\
\breve{v}\left(\mathbf{x}_{0}, t\right)
\end{array}\right] \triangleq \frac{1}{t} \int_{0}^{t} d t^{\prime} \mathbf{V}\left(\mathbf{x}_{0}, t^{\prime}\right)=\frac{\boldsymbol{\phi}\left(\mathbf{x}_{0}, t\right)-\mathbf{x}_{0}}{t},
$$

where $\mathbf{V}$ is the Lagrangian velocity. The authors then separate mesoelliptic areas - areas over which the tracer gradients turn while keeping their norm unchanged-and mesohyperbolic areas-areas over which the gradients increase or decreasedepending on the sign of the following criterion:

$$
Q_{\mathrm{Mez}}=\operatorname{det}\left(\nabla_{\mathbf{v}}^{\mathrm{T}}\right)\left[\operatorname{det}\left(\nabla \breve{\mathbf{v}}^{\mathrm{T}}\right)-\frac{4}{t^{2}}\right]
$$

Working with the mesochronic velocity $\breve{\mathbf{v}}$ or with the flow $\boldsymbol{\phi}$ is mathematically equivalent, expressed by the definition (2.5). The above criterion is thus similar in spirit to CauchyGreen-tensor-based analyses. Nevertheless, that criterion is frame dependent unlike Cauchy-Green-tensor-based metrics like FTLE or the growth rate $\alpha^{2}$ (Karrasch 2015; Hadjighasem et al. 2017). Consequently, the classification of trajectories proposed by Mezić et al. (2010) will unfortunately change under changes of a moving observer. To gain insight about physical features of mixing, the Cauchy-Green-tensor-based metrics shall thus be promoted.

Still, our growth rate $\alpha^{2}$ can be expressed with the mesochronic notations. In appendix $\mathrm{C}$, we rewrite the criterion as

$$
Q_{\mathrm{Mez}} t^{2}=\underbrace{\left(\partial_{x} \breve{u}-\partial_{y} \breve{v}\right)^{2}+\left(\partial_{y} \breve{u}+\partial_{x} \breve{v}\right)^{2}}_{=2(\alpha / t)^{2}}-\breve{\omega}^{2},
$$

where we introduce the mesochronic vorticity $\breve{\omega} \triangleq \nabla^{\perp} \cdot \breve{v}$ and $\nabla^{\perp} \triangleq\left(-\partial_{y}, \partial_{x}\right)^{\mathrm{T}}$ the orthogonal gradient operator in 2D. Note that the mesochronic vorticity is not the time-averaged vorticity along a trajectory and is frame dependent. The above derivation (2.7) then becomes reminiscent of the Okubo-Weiss criterion (Okubo 1970; Weiss 1991; Shivamoggi and van Heijst 2011). Indeed, it shows the competition between the strain and the rotation of the mesochronic velocity, encoded by $\alpha$ and $\breve{\omega}$, respectively. Moreover, according to (2.3), frame-dependent mesoelliptic regions are associated with zero growth rate $\alpha^{2}$, and expression (2.7) leads to $Q_{\mathrm{Mez}}=-(\varpi / t)^{2} \leq 0$. From our proposed development, the frame dependency and interpretation of the stretching criterion (2.7) is thus better characterized.

\section{c. Folding}

Stretching occurs when two points, $\phi(\mathbf{x})$ and $\phi(\mathbf{x}+\delta \mathbf{x})$, become closer or diverge, strengthening tracer gradients. This property is again naturally encoded in the Cauchy-Green tensor:

$$
\|\boldsymbol{\phi}(\mathbf{x}+\boldsymbol{\delta} \mathbf{x})-\boldsymbol{\phi}(\mathbf{x})\|^{2} \approx\left\|\left(\nabla \boldsymbol{\phi}^{\mathrm{T}}\right)^{\mathrm{T}} \boldsymbol{\delta} \mathbf{x}\right\|^{2}=\boldsymbol{\delta} \mathbf{x}^{\mathrm{T}} \nabla \boldsymbol{\phi}^{\mathrm{T}}\left(\nabla \boldsymbol{\phi}^{T}\right)^{\mathrm{T}} \boldsymbol{\delta} \mathbf{x}
$$

Mixing can occur when folding is associated with stretching. Folding is thus associated with a three-point kinematic property. Indeed, at least three points-e.g., $\boldsymbol{\phi}(\mathbf{x}), \boldsymbol{\phi}(\mathbf{x}+\boldsymbol{\delta} \mathbf{x})$, and $\boldsymbol{\phi}(\mathbf{x}-\boldsymbol{\delta} \mathbf{x})$-are needed to represent a folding. First, the three points are separated by stretching, creating a filament. Then, the filament folds bringing the two opposite points $[\boldsymbol{\phi}(\mathbf{x}+\boldsymbol{\delta} \mathbf{x})$ and $\boldsymbol{\phi}(\mathbf{x}-\boldsymbol{\delta} \mathbf{x})$ ] closer again. This folding can trap an area having a distinct tracer value (squeezing), creating strong tracer gradients. The folding is encoded by the relative evolution of positions increments $[\boldsymbol{\phi}(\mathbf{x}+\boldsymbol{\delta} \mathbf{x})-\boldsymbol{\phi}(\mathbf{x})]$ and $[\boldsymbol{\phi}(\mathbf{x}-\boldsymbol{\delta x})-\boldsymbol{\phi}(\mathbf{x})]$.

In the next section, we will show that folding is a key aspect of tracer gradient strengthening, even with stationary Eulerian velocity. Indeed, the minimal requirement for folding to occur is the nonlinearity in space of that velocity field.

To recall, many mixing diagnostics exist in the literature (Hadjighasem et al. 2017). Most are stretching proxies. However, few methods exist to diagnose folding and its relation to stretching. For instance, Ma et al. (2016) directly measure folding of material lines through an analysis of their curvature variations. In the following, we provide new relationships to further relate stretching and gradient of the curvature of streamlines in the case of a slowly varying Eulerian velocity field.

\section{Approximations for coarse-scale observations}

\section{a. Decorrelation approximations}

Over the space, flows encompass several eddies, e.g., flows are not laminar, and the angle $\theta_{T_{0}}^{\boldsymbol{\phi}}$ appearing in (2.3) will take different values. If the flow gradients and the initial tracer gradients are not locally correlated, i.e., are oriented with various angles over the space, the variance of $\theta_{T_{0}}^{\boldsymbol{\phi}}$ will likely be large. For large enough areas, the distribution of $2 \theta_{T_{0}}^{\boldsymbol{\phi}}[2 \pi]$ over the space will then become close to a uniform law on $[0,2 \pi]$. In (2.3), the average over the space of the term $\cos \left(2 \theta_{T_{0}}^{\boldsymbol{\phi}}\right)$ will then become close to zero, and finally, the absence of salient alignments between the initial tracer gradient and the flow gradient (i.e., cross correlations between the initial tracer and the flow) leads to

$$
\frac{\overline{\|\nabla T\|^{2}}}{\| \overline{\left\|\nabla T_{0}\right\|^{2}}} \approx 1+\overline{\alpha^{2}}
$$

On average, the tracer gradients will thus always increase by stretching. Welander (1955) already illustrated the process. The tracer - a dye patch or an oil spill introduced at time $t=$ 0 -is completely passive. In this case, the tracer and the flow are locally completely misaligned (i.e., uncorrelated in the above sense), and the initial structure of the tracer is quickly stretched and folded to fill a broad range of scales.

In contrast, geophysical tracers are generally correlated, i.e., more or less aligned, with some flow-dependent directions. Even passive tracers may be correlated to the flow due to their long-lasting patterns induced by past advection history. The effect of those correlations is expressed by the angle $\theta_{T_{0}}^{\boldsymbol{\phi}}$ in the right-hand-side integrand of Eq. (2.3), which can be 
locally positive or negative. Accordingly, local correlations can restrict or reduce the emergence of locally enhanced strong gradients. Berti and Lapeyre (2014), Dencausse et al. (2014), and Rogé et al. (2015) applied Lagrangian advection method to passive and active tracers-SST or SSS - to possibly reconstruct finer-scale ocean tracer patterns. However, submesoscales and also some mesoscales of the initial tracer and of the flow used in these Lagrangian methods are often missing, especially using interpolated ocean products. This is likely to strongly reduce local alignments. So, (3.1) generally holds to predict a strengthening of mesoscale and submesoscale tracer gradients, which is confirmed in the results of Berti and Lapeyre (2014), Dencausse et al. (2014), and Rogé et al. (2015).

\section{b. Time dependency}

In line with the geostrophy assumption used to estimate velocities from satellite sea surface height measurements, the resulting large-scale Eulerian ocean flows are also slowly varying (with characteristic time scale of about 10 days). The Lagrangian downscaling methods of Dencausse et al. (2014) and Rogé et al. (2015) aimed to consistently downscale tracer fields, SST and SSS, respectively. These methods use quasistationary large-scale velocity fields when applying Lagrangian-advection schemes over one or two weeks. For ocean scales of order $100 \mathrm{~km}$, a typical velocity correlation time is about 1 month. Hence, for such spatial scales, the flow field can well be assumed stationary. This assumption determines a specific form for the flow. In particular, the flow is not chaotic (Thiffeault 2004).

Furthermore, we will show that for such a flow the time dependency analysis of the mixing can be separated between two typical classes: open straight streamlines and closed curved streamlines. For both cases, the growth rate $\alpha^{2}$ is proportional to $t^{2}$.

\section{1) LOCALLY UNIFORM SHEAR}

Let us first focus on locally straight streamlines (i.e., streamlines with zero curvature). In such a case, the strengthening of tracer gradients results from a velocity shear, similarly to usual infinitesimal-time stretching. We denote by $x$ the local axis of the straight streamline and by $u=(\mathbf{v} /\|\mathbf{v}\|) \cdot \mathbf{v}=\|\mathbf{v}\|$, the velocity component on this direction. The divergence-free assumption imposes

$$
\partial_{x} u=\nabla \cdot \mathbf{v}=0
$$

Since the Eulerian velocity is stationary, the Lagrangian velocity is stationary as well:

$$
\frac{d \mathbf{V}}{d t}\left(\mathbf{x}_{0}, t\right)=\frac{d}{d t}\left\{\mathbf{v}\left[\boldsymbol{\phi}\left(\mathbf{x}_{0}, t\right)\right]\right\}=\left(u \partial_{x} u \frac{\mathbf{v}}{\|\mathbf{v}\|}\right)\left[\boldsymbol{\phi}\left(\mathbf{x}_{0}, t\right)\right]=0,
$$

and the flow simplifies to

$$
\boldsymbol{\phi}\left(\mathbf{x}_{0}, t\right)=\mathbf{x}_{0}+\int_{0}^{t} d t^{\prime} \mathbf{V}\left(\mathbf{x}_{0}, t^{\prime}\right)=\mathbf{x}_{0}+\mathbf{v}\left(\mathbf{x}_{0}\right) t=\mathbf{x}_{0}+\left[\begin{array}{c}
u\left(\mathbf{x}_{0}\right) t \\
0
\end{array}\right]
$$

This so-called ballistic regime is superdiffusive (Vallis 2006; Falkovich et al. 2001). Taking the gradient of the above expression with the divergence-free condition (3.2), the stretching rate reads

$$
\alpha^{2} \triangleq \frac{1}{2}\left\|\nabla \boldsymbol{\phi}^{\mathrm{T}}\right\|^{2}-1=\left(\frac{t}{\tau_{s}}\right)^{2}
$$

with the shearing time $\tau_{s}$

$$
1 / \tau_{s}=\frac{1}{\sqrt{2}} \partial_{y} u
$$

For a computation independent of any specific local axis $x$, we rewrite

$$
1 / \tau_{s}=\frac{1}{\sqrt{2}} \frac{\mathbf{v}^{\perp}}{\left\|\mathbf{v}^{\perp}\right\|} \cdot \nabla\|\mathbf{v}\|
$$

with $\mathbf{v}^{\perp}$ the $\pi / 2$ rotation of $\mathbf{v}$.

\section{2) STATIONARY CONVECTIVE CELLS}

Close to rotating eddies, streamlines are often closed or at least curved, and the previous development cannot be applied. Let us focus on closed streamlines. Since the flow is incompressible, fluid parcels cannot accumulate. Therefore, those streamlines define loops, called stationary convective cells (Falkovich et al. 2001), where fluid parcels rotate periodically. Accordingly, the flow and thus the Lagrangian velocity are periodic and the flow is called subdiffusive (Vallis 2006; Falkovich et al. 2001). This geometry can nevertheless create a strong stretching effect in finite time. Indeed, two concentric closed streamlines can define Lagrangian loops associated with different rotation periods. Rotation after rotation, a fluid parcel on the fastest loop will deviate from its initial neighboring parcel on the slowest loop. This differential rotation thus creates stretching. Moreover, it also induces folding. A filament distributed perpendicular to streamlines will be deformed by the continuous differential rotation. After a finite time, the filament will wrap around the convective cell creating spirals. Lehahn et al. (2007) illustrate a similar process with the action of stable and unstable manifolds on phytoplankton patches. In the same idea, Haller et al. (2016), and Haller (2016) propose two Lagrangian mixing diagnoses - a variant of the polar rotation angle (PRA) and the Lagrangian-averaged vorticity deviation (LAVD)-defining coherent sets with points having similar rotations.

To express the stretching induced by those convective cells, the flow is written as follows:

$$
\boldsymbol{\phi}\left(\mathbf{x}_{0}\right)=\boldsymbol{\phi}\left(\mathbf{x}_{0}, t\right) \approx \mathbf{x}_{0}+\mathbf{g}\left[\mathbf{x}_{0}, f\left(\mathbf{x}_{0}\right) t\right],
$$

where $\mathbf{g}$ is 1 - periodic with respect to its second variable and $f\left(\mathbf{x}_{0}\right)$ is the local temporal frequency. For a point initially on $\mathbf{x}_{0}$ in a closed streamline $\mathcal{C}$, the trajectory $t \mapsto \boldsymbol{\phi}\left(\mathbf{x}_{0}, t\right)$ runs from $\mathbf{x}_{0}$ to $\mathbf{x}_{0}$ through a path $\mathcal{P}$ embedded in $\mathcal{C}$ with a temporal period $1 / f\left(\mathbf{x}_{0}\right)$ defined by 


$$
\frac{1}{f\left(\mathbf{x}_{0}\right)}=\int_{0}^{\frac{1}{f\left(\mathbf{x}_{0}\right)}} d t=\int_{\mathcal{P}} \frac{d l}{\|\mathbf{v}\|}=\oint_{\mathcal{C}} \frac{\mathbf{v}}{\|\mathbf{v}\|^{2}} \cdot d \mathbf{l}
$$

The last integral only depends on the streamline $\mathcal{C}$ and not on the precise initial condition $\mathbf{x}_{0}$, and the local frequency inherits from the same invariance. Besides, the points $\mathbf{x}_{0}$ and $\phi\left(\mathbf{x}_{0}, t\right)$ are on the same streamline, and thus

$$
f\left[\boldsymbol{\phi}\left(\mathbf{x}_{0}, t\right)\right]=f\left(\mathbf{x}_{0}\right) .
$$

This frequency can be approximated by a local angular velocity $\dot{\theta}$, estimated using the streamline curvature, denoted $1 / R$, as

$$
f \approx \frac{\dot{\theta}}{2 \pi} \approx \frac{\|\mathbf{v}\|}{2 \pi R}=\frac{1}{2 \pi}\left[(\mathbf{v} \cdot \nabla) \frac{\mathbf{v}}{\|\mathbf{v}\|}\right] \cdot \frac{\mathbf{v}^{\perp}}{\left\|\mathbf{v}^{\perp}\right\|}
$$

In practice, the exact formula (3.9) can be difficult to evaluate numerically, and we will instead use the above approximation. In the following derivation, however, we keep the exact definition (3.9). In particular, we still assume the frequency invariance along the streamline (3.10). The first variable of $\mathbf{g}$ encodes the spatial dependency of the loop (vectorial) amplitudes. Note that the model (3.8) is very general, only assuming periodicity of Lagrangian trajectories. It helps to partially decouple flow variations associated with different streamlines (i.e., different local frequencies $f$ ) and flow variations associated with different temporal phase shifts along the streamline (i.e., different times $t$ ). To some extent, this second type of variation can be understood as different initial conditions in the same streamline, due to the periodicity assumption. Similar decomposition ideas were proposed by Thiffeault (2004) for chaotic (nonperiodic) flows.

In appendix $\mathrm{D}$, it is shown that time dependency of the growth rate in the final grid (points $\mathbf{x}$ ) reads

$$
\alpha^{2}\left[\boldsymbol{\phi}^{-1}(\mathbf{x}, t), t\right]=\left[\frac{t}{\tau_{f}(\mathbf{x})}\right]^{2}
$$

with the folding time $\tau_{f}$

$$
1 / \tau_{f}=\frac{\|\nabla f|\|\mid \mathbf{v}\|}{\sqrt{2} f}
$$

As (3.1) only involves the spatial average of $\alpha^{2}$, we can further simplify the model by spatial integration. Indeed, integrating Eq. (3.12) over a specific domain $\Omega_{f}$, we obtain with the variable change defined by the incompressible flow:

$$
\int_{\Omega_{f}} d \mathbf{x}_{0} \alpha^{2}\left(\mathbf{x}_{0}, t\right)=\int_{\boldsymbol{\phi}\left(\Omega_{f}\right)} d \mathbf{x} \alpha^{2}\left[\boldsymbol{\phi}^{-1}(\mathbf{x}, t), t\right]=t^{2} \int_{\boldsymbol{\phi}\left(\Omega_{f}\right)} \frac{d \mathbf{x}}{\tau_{f}^{2}(\mathbf{x})}
$$

The subspace $\Omega_{f}$ is a subset of $\Omega$ where the concept of wrapping convective cells is relevant. Since we consider closed streamlines, we assume that $\boldsymbol{\phi}\left(\Omega_{f}\right)=\Omega_{f}$. This subspace will be properly defined in the following.

\section{3) GLOBAL TIME DEPENDENCY}

To combine the folding time $\tau_{f}$ and the shearing time $\tau_{s}$, a local stretching time $\tau$ is defined depending upon the local streamline curvature:

$$
\tau\left(\mathbf{x}_{0}\right) \triangleq\left\{\begin{array}{c}
\tau_{f}\left(\mathbf{x}_{0}\right) \text { if } R\left(\mathbf{x}_{0}\right) \leq \frac{L}{2} \\
\tau_{s}\left(\mathbf{x}_{0}\right) \text { if } R\left(\mathbf{x}_{0}\right)>\frac{L}{2}
\end{array},\right.
$$

where $1 / R\left(\mathbf{x}_{0}\right)$ is the streamline curvature on $\mathbf{x}_{0}$, and $L$ the average diameter of a vortex. Following the previous models of shearing and folding, the stretching rate becomes

$$
\alpha=\frac{t}{\tau} .
$$

Where gradients are created by uniform shears, streamlines are straight, the curvature $1 / R$ is small, and $\tau=\tau_{s}$, whereas, at locations where gradients are strengthened by wrapping, the curvature is large and $\tau=\tau_{f}$.

To estimate the average eddy diameter $L$, a toy approximation is used to locally define the velocity:

$$
\mathbf{v}=U\left[\begin{array}{l}
\cos \left(\frac{2 \pi}{\lambda} x\right) \sin \left(\frac{2 \pi}{\lambda} y\right) \\
\sin \left(\frac{2 \pi}{\lambda} x\right) \cos \left(\frac{2 \pi}{\lambda} y\right)
\end{array}\right]
$$

and the eddy diameter is identified to the size of the convective cell:

$$
L=\frac{\lambda}{2}=\left(\frac{6 \pi^{2} \overline{\|\mathbf{v}\|^{2}}}{\left\|\nabla \mathbf{v}^{\mathrm{T}}\right\|^{2}}\right)^{1 / 2}
$$

We shall then use this diameter estimator in the general case. The condition (3.15) further defines a space partition $\Omega=\Omega_{f} \cup \Omega_{s}$ to integrate the growth rate $\alpha^{2}$ :

$$
\overline{\alpha^{2}}=\left(\frac{t}{\tau_{G}}\right)^{2} \text { with } \frac{1}{\tau_{G}^{2}} \triangleq \overline{\left(\frac{1}{\tau^{2}}\right)}=\frac{1}{S}\left[\int_{\Omega_{f}} \frac{d \mathbf{x}}{\tau_{f}^{2}(\mathbf{x})}+\int_{\Omega_{s}} \frac{d \mathbf{x}_{0}}{\tau_{s}^{2}\left(\mathbf{x}_{0}\right)}\right],
$$

where $\quad \Omega_{f} \triangleq[\mathbf{x} \in \Omega \mid R(\mathbf{x}) \leq(L / 2)] \quad$ and $\quad \Omega_{s} \triangleq\left[\mathbf{x}_{0} \in \Omega \mid R\left(\mathbf{x}_{0}\right)>\right.$ $L / 2]$. Again, $\boldsymbol{\phi}\left(\Omega_{f}\right)=\Omega_{f}$ is assumed because the flow maps closed streamlines onto themselves. In the following, $\tau_{G}$ is referred as the global stretching time.

The model (3.19) together with the folding and shearing time definitions (3.13)-(3.7) thus specify a global Eulerian estimate of finite-time stretching. Unlike usual Lagrangian diagnoses, such as FTLE and finite-size Lyapunov exponents (FSLE) (d'Ovidio et al. 2009), the proposed global model does not require any integration of the flow. Finally, according to (3.1), the evolution law (3.19) determines the tracer gradient norm:

$$
\frac{\overline{\|\nabla T\|^{2}}}{\overline{\left\|\nabla T_{0}\right\|^{2}}} \approx 1+\left(\frac{t}{\tau_{G}}\right)^{2} .
$$




\section{Tracer spectral tail}

It has long been realized (Batchelor 1959) that the general increase of gradients of $T$, during the stirring action of an underlying flow field, is a consequence of local misalignments between tracer isolines and the velocity vector field, leading to a transfer of tracer variance from low wavenumber Fourier components to high ones. Mixing will thus be associated to strengthening processes acting on the tracer smallest scales. Mixing shall thus be characterized in the spectral domain, especially its high-wavenumber part. Overall Eulerian diagnostics of the tracer gradients norm evolution in the spatial domain must then be related to spectral diagnoses. In this section, this link is demonstrated.

After preliminary results related to tracer moments, we first derive a Gaussian approximation for the evolution of the spectral tail, assuming spatial smoothness. This approximation is then applied to initial and advected tracers. Finally, we propose an alternative development for self-similar spectra.

Because of the incompressiblity constraint $\left[\operatorname{det}\left(\nabla \boldsymbol{\phi}^{\mathrm{T}}\right)=1\right]$, as all scales are assumed to be resolved, and since the molecular diffusion is ineffective on the length and time scales of interest, there are no overlays of fluid parcels and no dilution of their properties. Each fluid parcel conserves its tracer value while it is advected. Therefore, mean and variance, $\bar{T}$ and $\overline{(T-\bar{T})^{2}}$, are conserved. As a consequence, we will assume without loss of generality that the tracer is centered.

\section{a. Locally smooth scalar approximation}

First, let us consider the covariance of a smooth scalar $q$, for small spatial distance $\|\boldsymbol{\delta} \mathbf{x}\|$. The scalar field $q$ will represent here the initial tracer $T_{0}$ or the advected tracer $T$. We will assume it is twice differentiable, which is a strong assumption on the scalar regularity. Yet, for tracers measured at mesoscales and reinterpolated on a submesoscale spatial grid, this assumption safely applies. Accordingly, the covariance of $q-$ denoted $\gamma_{q}$-is 4 times differentiable near the origin 0 and its Taylor expansion reads

$$
\begin{aligned}
\gamma_{q}(\boldsymbol{\delta} \mathbf{x}) & \triangleq \frac{1}{S} \int_{\Omega} d \mathbf{x} q(\mathbf{x}) q(\mathbf{x}+\boldsymbol{\delta} \mathbf{x}) \\
& =\overline{q^{2}}+\frac{1}{2} \boldsymbol{\delta} \mathbf{x}^{\mathrm{T}} \mathbf{H}_{\gamma_{q}}(0) \boldsymbol{\delta} \mathbf{x}+\underset{\boldsymbol{\delta} \mathbf{x} \rightarrow 0}{o}\left(\|\boldsymbol{\delta} \mathbf{x}\|^{3}\right) \\
& =\overline{q^{2}} \exp \left[-\frac{1}{2} \boldsymbol{\delta} \mathbf{x}^{\mathrm{T}}\left[\frac{-\mathbf{H}_{\gamma_{q}}(0)}{\overline{q^{2}}}\right] \boldsymbol{\delta} \mathbf{x}\right]+\underset{\|\boldsymbol{\delta} \mathbf{x}\| \rightarrow 0}{o}\left(\|\boldsymbol{\delta} \mathbf{x}\|^{3}\right)
\end{aligned}
$$

where $\mathbf{H}_{\gamma_{q}}$ denotes the Hessian of the covariance $\gamma_{q}$. This Gaussian covariance approximation - valid near the origin 0 only-results from the absence of infinitely small-scale structures in the reinterpolated field $q$. This approximation is not instructive over longer correlation distances (e.g., mesoscales), which often exhibit physical self-similar structures and gradient singularities. Typically, in an isotropic case and for intermediate values of spatial increments norms $\|\boldsymbol{\delta} \mathbf{x}\|$, we would have $\gamma_{q}(\boldsymbol{\delta} \mathbf{x}) \approx \overline{q^{2}}-C\|\boldsymbol{\delta} \mathbf{x}\|^{\zeta-1}$ with $\zeta<3$. This would correspond to an omnidirectional spectrum proportional to $\kappa^{-\zeta}$ for intermediate wavenumbers $\kappa$ and a diverging tracer gradient variance $\overline{\|\nabla q\|^{2}}=+\infty$. Here, we focus on the extremely local behavior of the reinterpolated tracer, which can be well approximated by the above Gaussian covariance with $-\overline{q^{2}} \mathbf{H}_{\gamma_{q}}^{-1}(0)$ as squared correlation lengths. Note, the evolution of the Hessian $\mathbf{H}_{\gamma_{q}}^{-1}(0)$ is difficult to characterize whereas its trace is simple and reads $-\overline{\|\nabla q\|^{2}}$ (which is here finite and well defined, see appendix E). To let this gradient norm explicitly appear instead of the covariance Hessian, we consider tracer statistics averaged over angles. In Fourier space, the omnidirectional spectrum is defined as follows:

$$
\tilde{\Gamma}_{q}(\kappa) \triangleq \kappa \oint_{[0,2 \pi]} d \theta_{\mathbf{k}}|\hat{q}(\mathbf{k})|^{2},
$$

where the hat denotes spatial Fourier transform, $\mathbf{k}=$ $\kappa\left[\cos \left(\theta_{k}\right) \sin \left(\theta_{k}\right)\right]^{\mathrm{T}}$ is the wavevector and $\kappa=\|\mathbf{k}\|$ the wavenumber. In the appendix E, we show that the local approximation (4.3) leads to

$$
\tilde{\Gamma}_{q}(\kappa) \underset{\kappa \rightarrow \infty}{\sim} C_{q} \exp \left(-\frac{1}{2} L_{q}^{2} \kappa^{2}\right)
$$

where

$$
L_{q}^{2}=\frac{\overline{q^{2}}}{\|\nabla q\|^{2}} \text { and } C_{q}=2\left[\frac{(2 \pi)^{3}\left(\overline{q^{2}}\right)^{3}}{\overline{\|\nabla q\|^{2}}}\right]^{1 / 2} .
$$

Similarly to the local covariance approximation (4.3), the above result is valid for $\kappa \gg 1 / L_{q}$ or at least $\kappa>1 / L_{q}$. The absence of infinitely small-scale structures implies a spectral roll-off at the highest wavenumbers. Equation (4.5) approximates this roll-off by a Gaussian decay and (4.6) determines the position of that roll-off.

\section{b. Tracer spectral tail evolution}

We now apply the tail approximation (4.5) to both the initial tracer $T_{0}$ and the advected tracer $T$. Here again, $T_{0}$ and $T$ are not the real full-scale ocean tracers, but correspond instead to coarse-scale observations under a fictitious smooth surface current advection. Because a fine interpolation grid is used for both $T_{0}$ and $T$, the spectrum Gaussian roll-off approximation (4.6) is valid for both fields and yields

$$
\begin{aligned}
& \tilde{\Gamma}_{T}(\kappa) \underset{\kappa \rightarrow \infty}{\sim} \tilde{\Gamma}_{T_{0}}(\kappa) \frac{C_{T}}{C_{T_{0}}} \exp \left[-\frac{1}{2}\left(L_{T}^{2}-L_{T_{0}}^{2}\right) \kappa^{2}\right] \\
& \underset{\kappa \rightarrow \infty}{\sim} \tilde{\Gamma}_{T_{0}}(\kappa)\left(\overline{\frac{\left\|\nabla T_{0}\right\|^{2}}{\|\nabla T\|^{2}}}\right)^{1 / 2} \exp \left[\frac{1}{2} \overline{T_{0}^{2}}\left(\frac{1}{\overline{\left\|\nabla T_{0}\right\|^{2}}}-\frac{1}{\overline{\|\nabla T\|^{2}}}\right) \kappa^{2}\right],
\end{aligned}
$$

where the simplification in the last asymptotic equivalence follows from the variance conservation. As discussed in section 3a, if the 


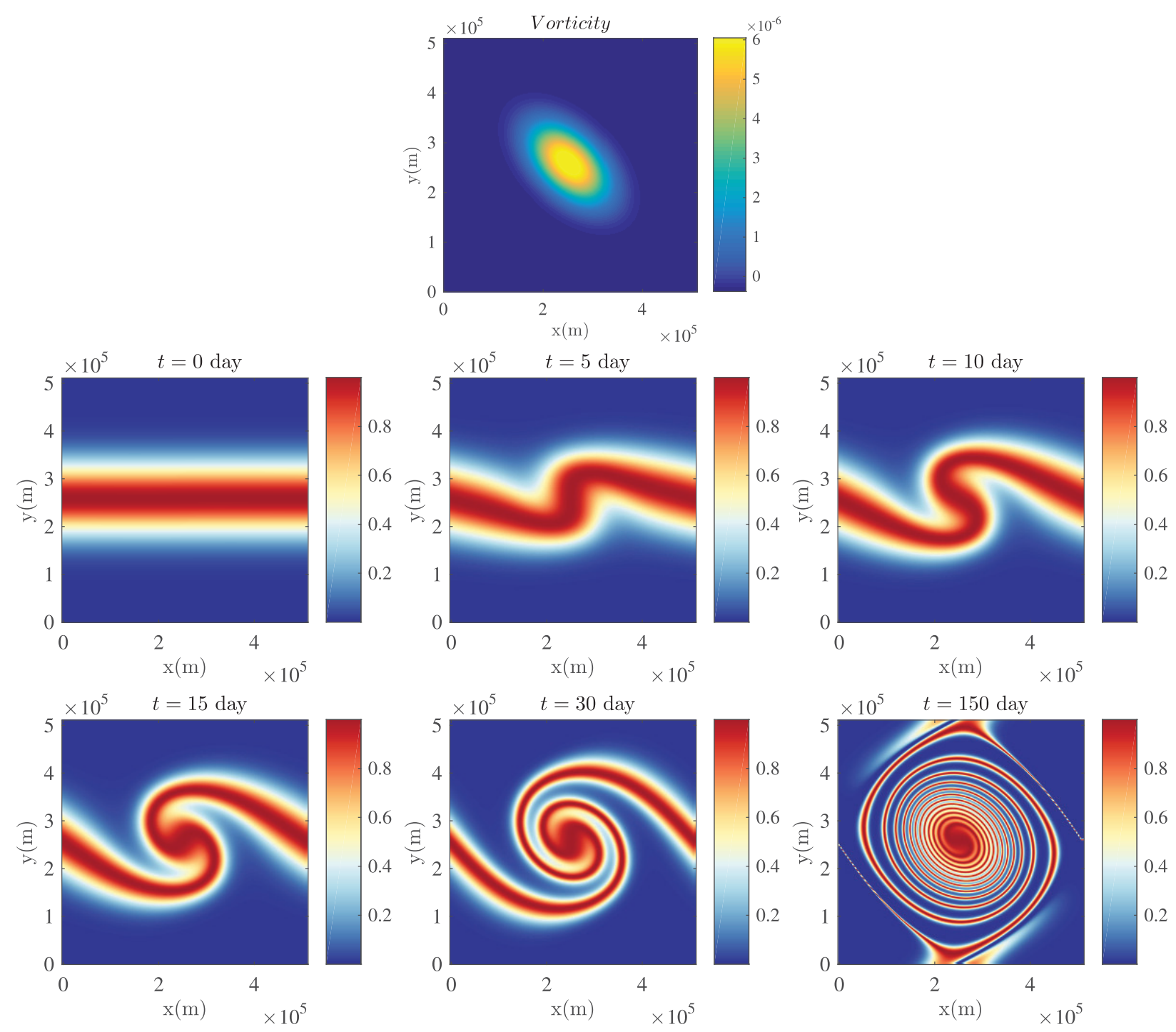

FIG. 1. (top) Advecting vorticity $\left(\mathrm{s}^{-1}\right)$ of the toy model and (middle),(bottom) tracer (dimensionless) advected using a backward Lagrangian method at time $t=0,5,10,15,30$, and 150 days.

initial tracer $T_{0}$ and the flow display local misalignments, the tracer gradients strengthen: $\overline{\|\nabla T\|^{2}}>\overline{\left\|\nabla T_{0}\right\|^{2}}$ (i.e., $L_{T}<L_{T_{0}}$ ) and by (4.8) the tracer spectral tail raises. Using the estimate (4.17), a final expression is derived:

$$
\tilde{\Gamma}_{T}(\kappa) \underset{\kappa \rightarrow \infty}{\sim} \tilde{\Gamma}_{T_{0}}(\kappa)\left[1+\left(\frac{t}{\tau_{G}}\right)^{2}\right]^{-1 / 2} \exp \left[\frac{1}{2} \frac{\overline{T_{0}^{2}}}{\overline{\left\|\nabla T_{0}\right\|^{2}}} \frac{\kappa^{2}}{1+\left(\frac{\tau_{G}}{t}\right)^{2}}\right] .
$$

It is thus possible to recover the initial spectrum tail by smoothing the advected tracer, as empirically noticed by Rogé et al. (2015) in their forward-backward Lagrangian advection method. Here, (4.9) provides a full parameterization of this low-pass Gaussian filter with squared length scale:

$$
\frac{\overline{T_{0}^{2}}}{\left\|\nabla T_{0}\right\|^{2}} \frac{1}{1+\left(\frac{\tau_{G}}{t}\right)^{2}}
$$

The multiplicative constant of (4.9) decreases with the advection time $t$. Asymptotically, we have

$$
\left(\overline{\frac{\left\|\nabla T_{0}\right\|^{2}}{\|\nabla T\|^{2}}}\right)^{1 / 2} \approx\left[1+\left(\frac{t}{\tau_{G}}\right)^{2}\right]^{-1 / 2}=\left\{\begin{array}{ll}
1 & \text { if } t \ll \tau_{G} \\
\frac{\tau_{G}}{t} & \text { if } t \gg \tau_{G}
\end{array} .\right.
$$

Note that this multiplicative constant does not provide information on large-scale tracer structures because we here rely on spectrum roll-off approximations. This constant is proportional to the advected tracer spectrum roll-off amplitude $C_{T}$. 

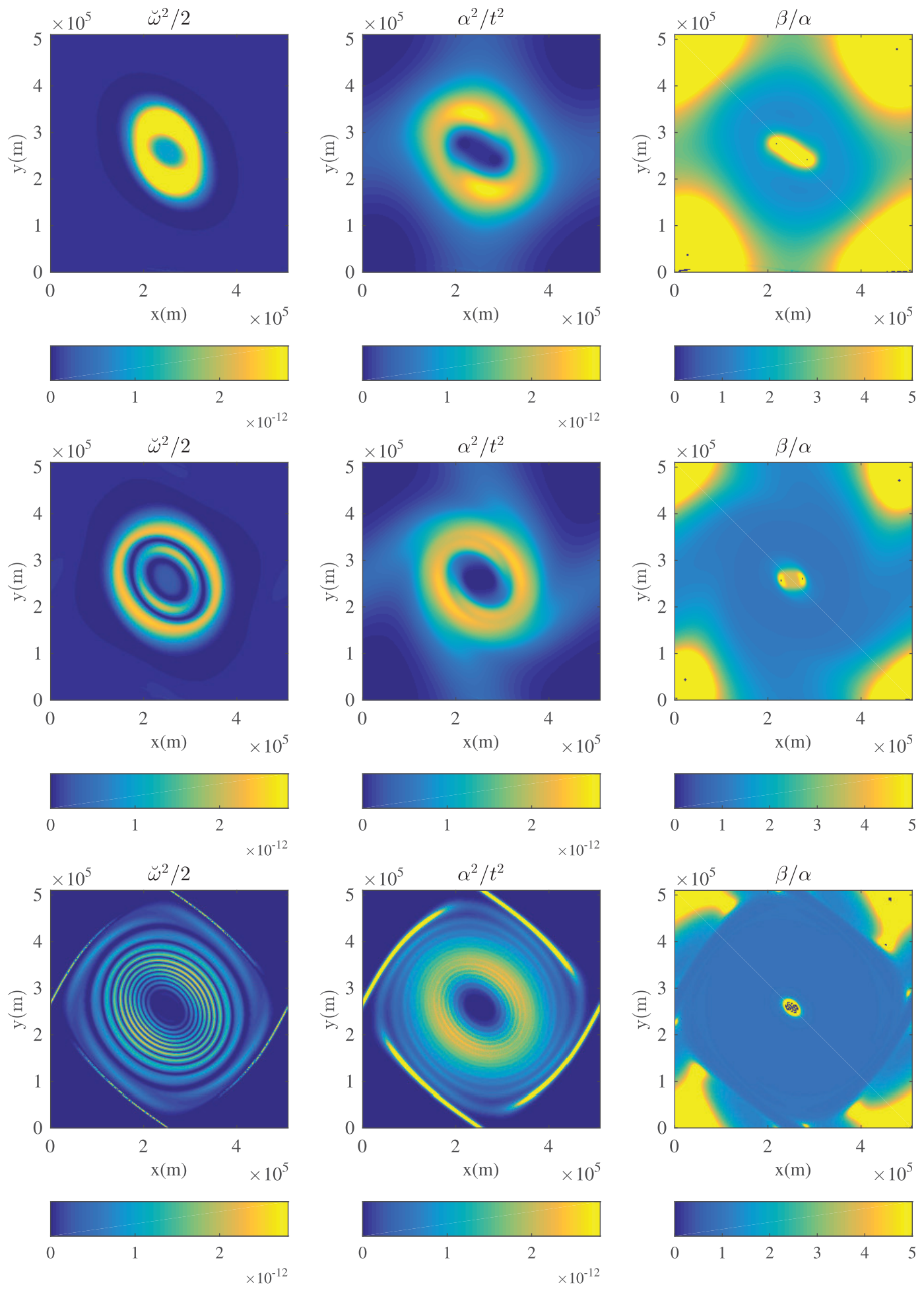

FIG. 2. Values of (left) $\breve{\omega}^{2} / 2\left(\mathrm{~s}^{-2}\right)$, (center) $(\alpha / t)^{2}(\alpha / t)^{2}\left(\mathrm{~s}^{-2}\right)$, and (right) the ratio of $\beta / \alpha$ (dimensionless) in the initial grid (points $\mathbf{x}_{0}$ ) at time (from top to bottom) $t=15,30$, and 150 days for the toy model. 

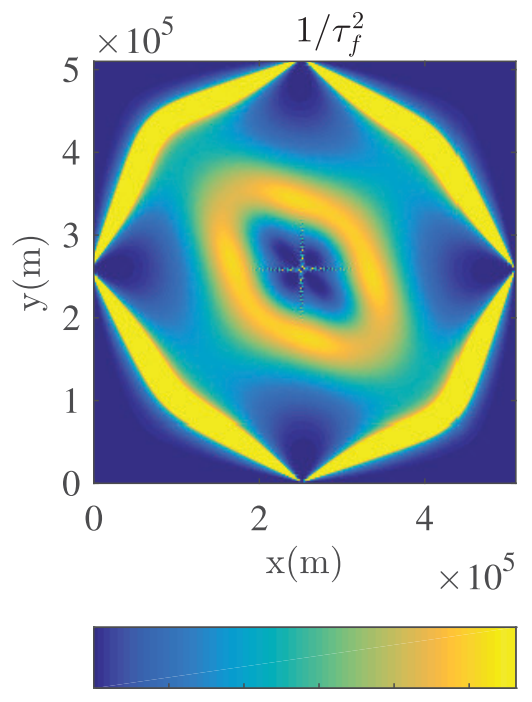

$\begin{array}{lllll}0.5 & 1 & 1.5 & 2 & 2.5\end{array}$ $\times 10^{-12}$
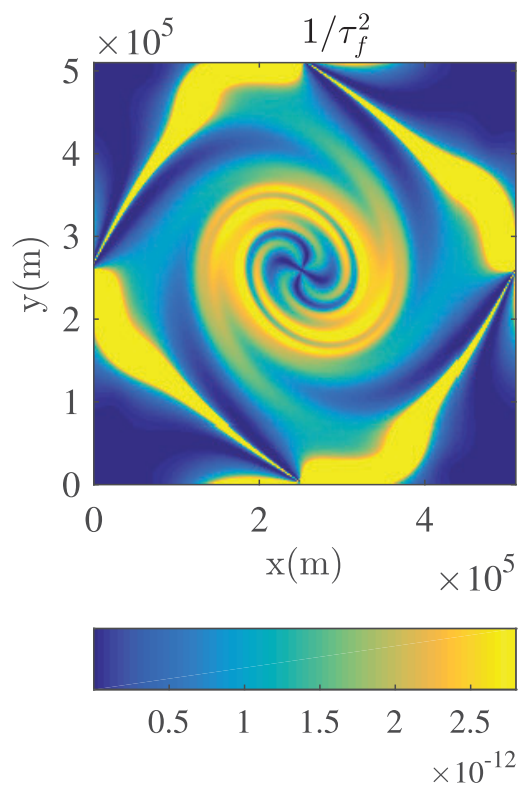
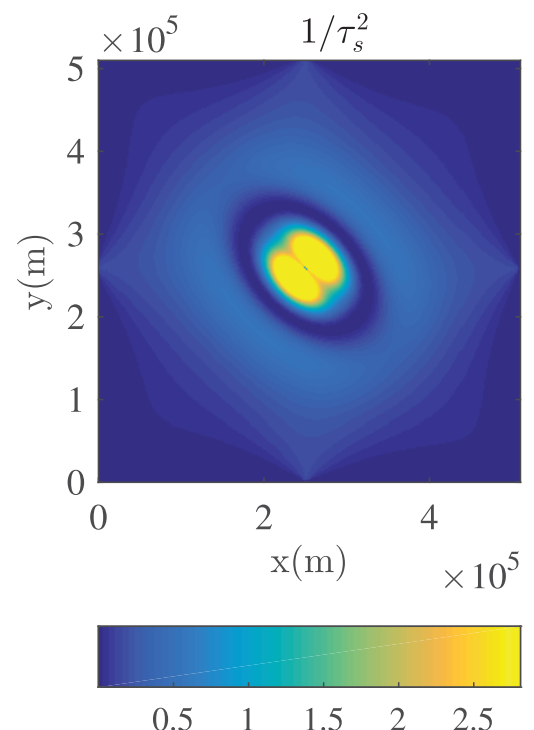
$\times 10^{-12}$
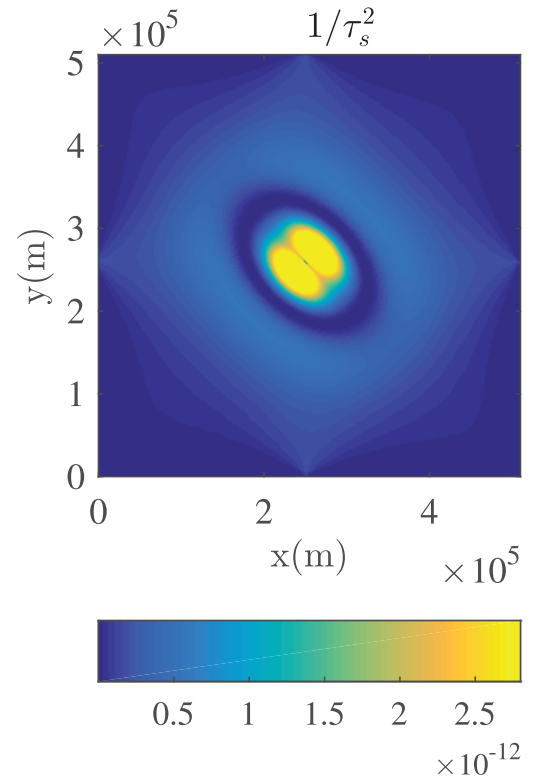
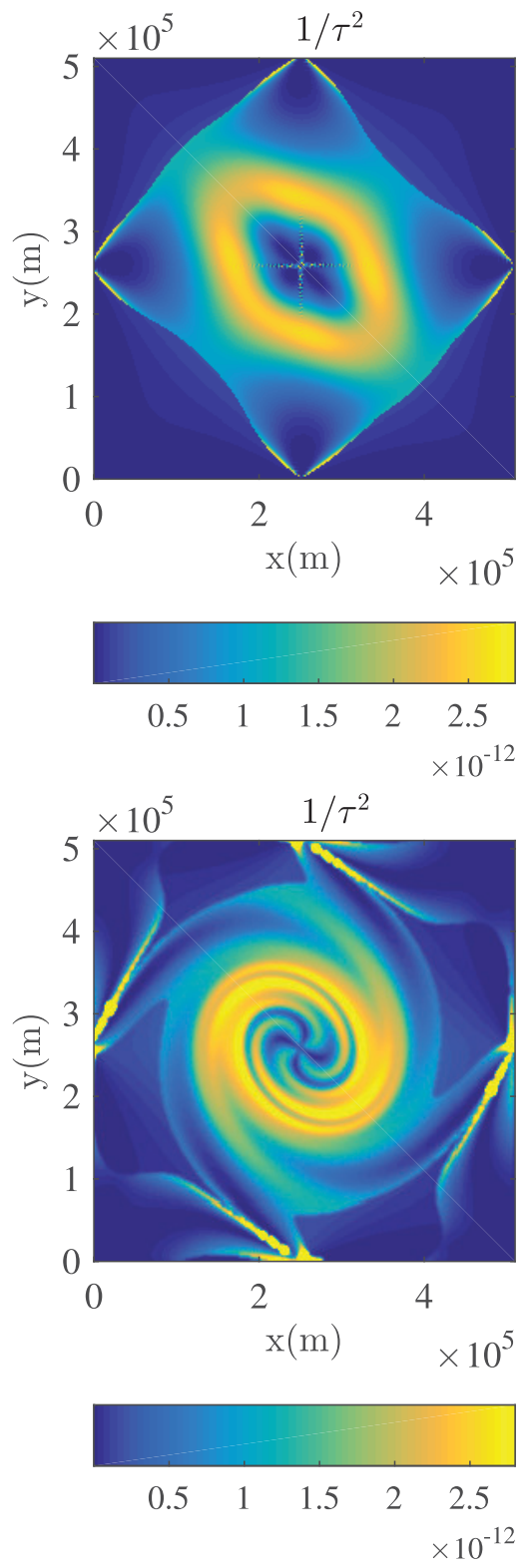

FIG. 3. Squared inverse of the folding time in the (top left) final grid (points $\mathbf{x}$ ) and (bottom left) initial grid (points $\mathbf{x}_{0}$ ), (center) of the shearing time in the initial grid (points $\mathbf{x}_{0}$ ), and of the stretching time in the (top right) final grid (points $\mathbf{x}$ ) and (bottom right) initial grid (points $\mathbf{x}_{0}$ ) for the toy model. All plots are in $\mathrm{s}^{-2}$. To represent folding and stretching times in the initial grid, these fields were advected during 30 days.

Since both this amplitude and the squared length scale (4.10) decreases with time, the advected tracer spectrum roll-off continuously moves toward higher wavenumbers and lower spectrum values during the downscaling advection.

\section{c. A practical estimation of an effective horizontal eddy diffusivity}

Spectral fall-off of real oceanic tracers being relatively stable, the predicted changes under multiple advection operations shall thus be compensated. It can be hypothesized to result from the combined antagonist effects of the wellresolved, slow-varying, and large-scale velocity properties; of the unresolved, fast-varying, and likely small-scale velocities; and finally, of smaller-scale tracer structures. In other words, the smoother velocity component will tend to raise the highwavenumber part of the spectrum (4.9), while the fast-varying velocity and tracer components shall act to balance this rise. This last process can be simply accounted for by considering the introduction of an effective spatially uniform eddy diffusivity $\nu$. After an advection of $\Delta t$, this effect leads to multiply the spectrum by $\exp \left(-\nu \Delta t \kappa^{2}\right)$. To exactly balance the 

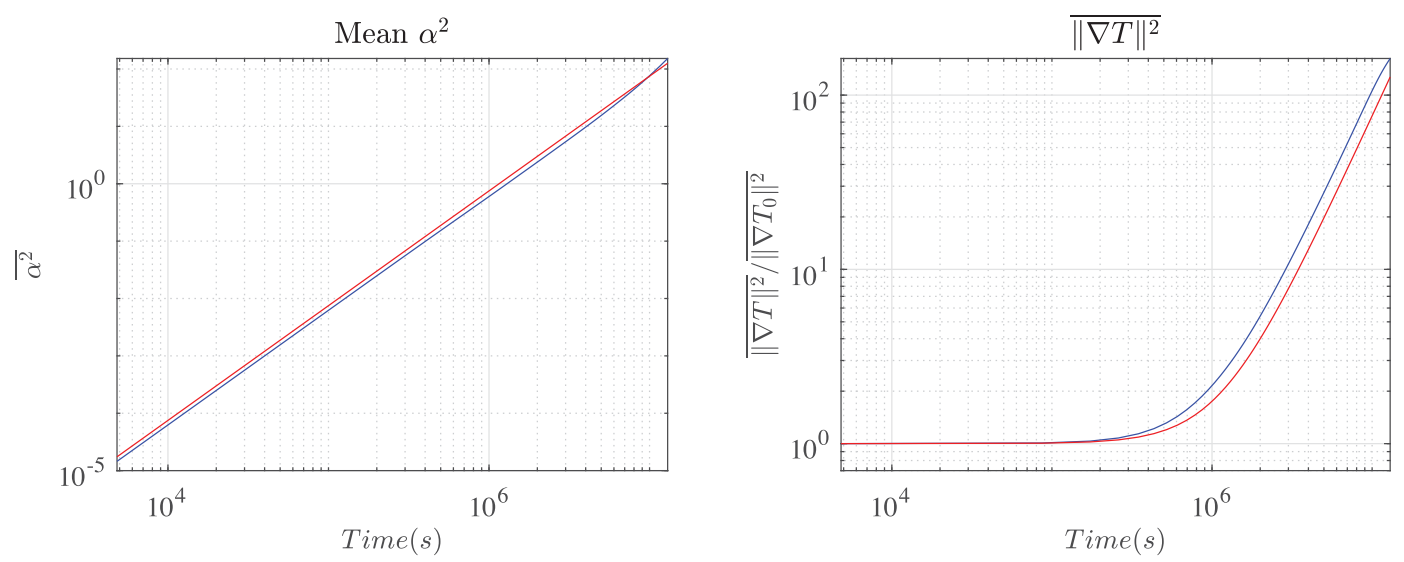

FIG. 4. (left) The averaged growth rate $\overline{\alpha^{2}}$ and (right) the averaged squared norm of tracer gradients for the toy model, both in log-log plot along time. The blue line is the real value and the red line our model.

expected high-wavenumber spectral rise (4.9) during $\Delta t$, and thus to keep the resulting advected tracer closer to its initial variance distribution over scales, the effective horizontal eddy diffusivity can thus be defined according to

$$
\nu=\frac{1}{2 \tau_{G}} \frac{\overline{T_{0}^{2}}}{\left\|\nabla T_{0}\right\|^{2}} c(t), \quad \text { with } c(t)=\frac{\frac{\Delta t}{\tau_{G}}}{\left[1+\left(\frac{\Delta t}{\tau_{G}}\right)^{2}\right]}=\left\{\begin{array}{ll}
\frac{\Delta t}{\tau_{G}} & \text { if } t \ll \tau_{G} \\
\frac{\tau_{G}}{\Delta t} & \text { if } \Delta t \gg \tau_{G}
\end{array} .\right.
$$

For small time step, $\Delta t$, the mixing is superdiffusive, whereas for large time step it is subdiffusive. The superdiffusive regime is the usual ballistic regime observed for small advection time $\Delta t$ (Vallis 2006; Falkovich et al. 2001) whereas the subdifffusive regime is less straightforward to understand. A spectrum roll-off-by definition-is concave, possibly to flatten for large advection time. Still, it cannot be convex. Additional advection steps eventually get less and less efficient at modifying the evolving form of the spectrum roll-off. Mathematically, the squared length scale of the low-pass Gaussian filter (4.10) cannot decrease less than its asymptotic value $\overline{T_{0}^{2}} / \overline{\left\|\nabla T_{0}\right\|^{2}}$. The eddy diffusivity $\nu$-being given by this (bounded) squared length scale by unit of timegets infinitely small for large times.

\section{d. Self-similar approximation for intermediate wavenumbers}

While the Gaussian approximation is useful to link advection and filtering operations, outside the roll-off and the planetary scales, spectra of geophysical tracer fields are more likely self-similar. Moreover, one may wish to target specific spectral slopes using the Lagrangian advection method. The following alternative form for the scalar spectrum is hence now considered:

$$
\tilde{\Gamma}_{q}(\kappa)=\left\{\begin{array}{c}
A\left(1+\frac{\kappa}{\kappa_{m}}\right)^{-\zeta} \text { if } \kappa \leq \kappa_{\infty} \\
0 \text { otherwise }
\end{array}\right.
$$

where $\kappa_{\infty}$ is set by the numerical resolution. For intermediate wavenumbers $\kappa_{m} \ll \kappa \ll \kappa_{\infty}$, the spectrum exhibits an inertial range $\tilde{\Gamma}_{q}(\kappa) \approx A\left(\kappa / \kappa_{m}\right)^{-\zeta}$. Unlike the previous local smoothness approximation, this inertial range is not an artifact of the field processing. It is a physical phenomenon induced by the advection by real oceanic currents, which also exhibit energy scale invariance. Accordingly,

$L_{q}^{2}=\frac{\overline{q^{2}}}{\overline{\|\nabla q\|^{2}}}$

$=\frac{\int_{0}^{\kappa_{\infty}}\left(1+\frac{\kappa}{\kappa_{m}}\right)^{-\zeta}}{\int_{0}^{\kappa_{\infty}} \kappa^{2}\left(1+\frac{\kappa}{\kappa_{m}}\right)^{-\zeta}}$

$$
=\frac{(\zeta-2)(\zeta-3)}{\kappa_{m}^{2}\left\{2-\left(1+\frac{\kappa_{\infty}}{\kappa_{m}}\right)^{1-\zeta}\left[(\zeta-1)(\zeta-2)\left(\frac{\kappa_{\infty}}{\kappa_{m}}\right)^{2}+2(\zeta-1) \frac{\kappa_{\infty}}{\kappa_{m}}+2\right]\right\}} .
$$

As long as the width of the inertial range, $\kappa_{\infty}-\kappa_{m}$, is large enough, the above function is strictly positive and continuous w.r.t. the spectral slope $\zeta$ for all $\zeta>1$.

Setting $q$ to the advected tracer $(q=T)$, the wavenumber $\kappa_{m}$ can encompass planetary length scales which do not vary much during the advection process. The resolution $\kappa_{\infty}$ is constant as well. So, a targeted spectral slope $\zeta$ conveniently 

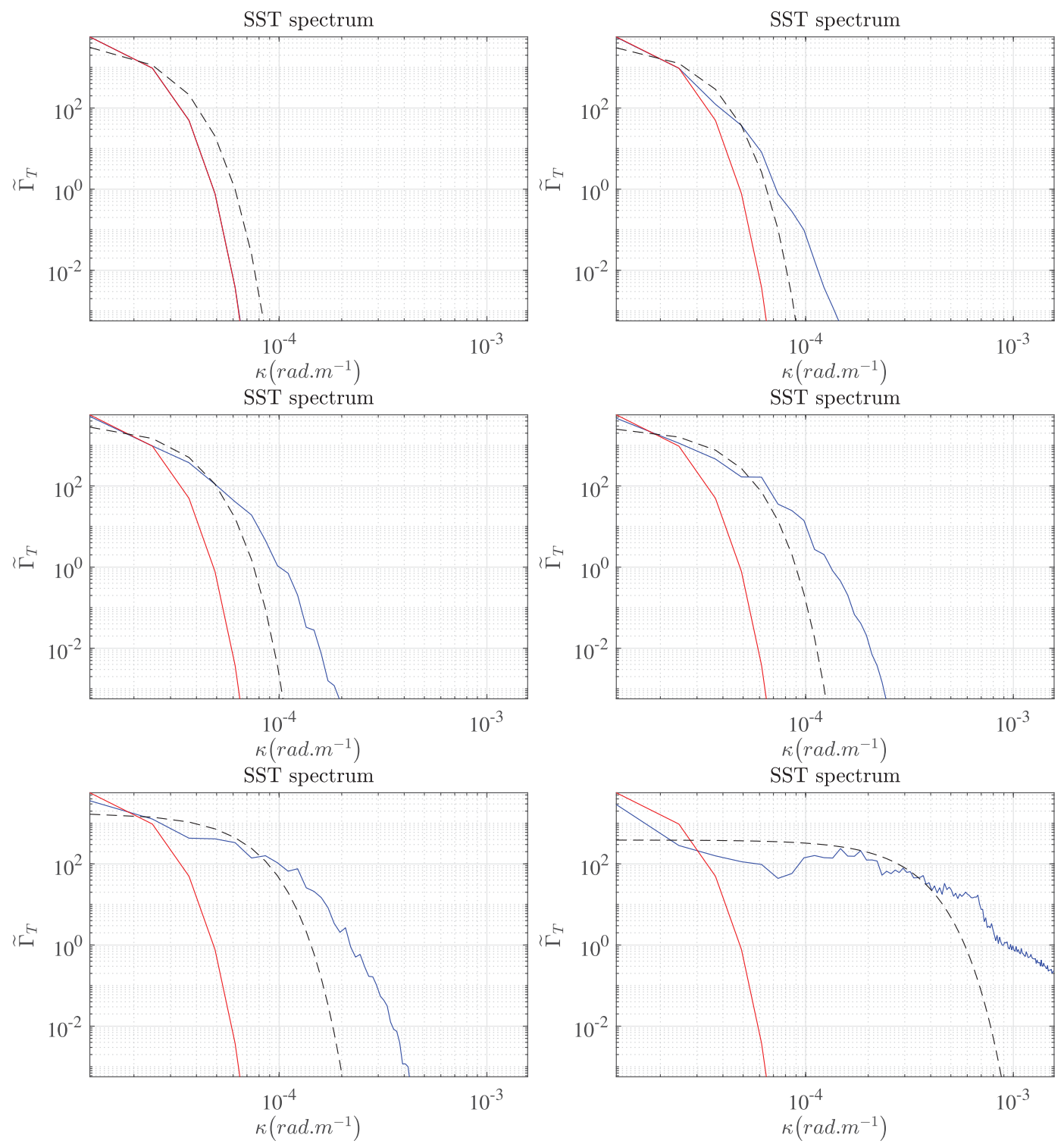

FIG. 5. Omnidirectional spectra before advection (red), after advection (blue), and its prediction using the Gaussian approximation (4.9) (dashed black line) for the toy model at $t=0,5,10,15,30$, and 150 days. The associated spatial fields are displayed in Fig. 1.

provides a length scale $L_{T}$ to be reached over a given advection time. Using (4.17), it can be estimated

$$
t=\tau_{G} \sqrt{\frac{1}{L_{T}^{2}(\zeta)} \frac{\overline{T_{0}^{2}}}{\left\|\nabla T_{0}\right\|^{2}}-1} .
$$

\section{Numerical results}

\section{a. Illustrative toy model}

These analytical developments can be first illustrated using a simplified toy model. We define an ellipsoidal eddy from the following vorticity field:

$$
\omega(\mathbf{x})=A_{\omega} \exp \left[-\frac{1}{2}\left(\frac{\|\mathbf{x}\|_{e}-r_{0}}{r_{\omega}}\right)^{2} \mathbf{1}_{\left\{\|\mathbf{x}\|_{e}>r_{0}\right\}}\right],
$$

with

$$
\|\mathbf{x}\|_{e}^{2}=[e(x+y)]^{2}+(x-y)^{2},
$$

an eccentricity $e=1.7, r_{0}=23.0 \mathrm{~km}, r_{\omega}=76.8 \mathrm{~km}$ and $A_{\omega}=$ $6.43 \times 10^{-6} \mathrm{~s}^{-1}$. The vorticity is constant at the ellipse center $\left(\left\|\mathbf{x}_{e}\right\| \leq r_{0}\right)$ and smoothly decreases to zero outside.

A large tracer filament is advected by that stationary velocity field using a backward Lagrangian advection (Fig. 1). For technical details, we refer to Berti and Lapeyre (2014) and Dencausse et al. (2014). The tracer progressively wraps, 
eventually creating infinitely long filaments. Following (3.19), an estimate of the global stretching time is 13.36 days. It roughly corresponds to half a rotation.

Figure 2 represents the spatial distribution of the time-normalized stretching rate $(\alpha / t)^{2}$, the factor $\beta / \alpha$ and the mesochronic vorticity $\breve{\omega}$ at several times. As found, the spatial distribution of $\alpha / t$ becomes nearly constant after one week only. This number is significant on the folding area, i.e., the border of the vortex. The ratio $\beta / \alpha=\sqrt{1+\left[2 /(\alpha / t)^{2}\right]\left(1 / t^{2}\right)}$ - which quantifies the significance of the orientation of tracer gradientdecreases with time in the mixing area. It stabilizes to its minimum value, say 1 , at $t \approx \tau_{G}$. The mesochronic vorticity is first concentrated in the center of the vortex. Then, after each global stretching time, a new ring of mesochronic vorticity adds to the mixing area.

Figure 3 displays the spatial distribution of the squared inverse of the folding time $\tau_{f}$, of the shearing time $\tau_{s}$, and of the stretching time $\tau$ for this toy model. Folding and stretching time are represented both in the initial grid $\left(\mathbf{x}_{0}\right)$ and in the advected grid $\left[\mathbf{x}=\boldsymbol{\phi}\left(\mathbf{x}_{0}, t\right)\right]$. This remapping on the initial grid is needed as the folding time is locally defined in the advected grid [see (3.12) and (3.14)]. For this remapping, we integrated the forward flow $\mathbf{x}_{0} \mapsto \boldsymbol{\phi}\left(\mathbf{x}_{0}, t\right)$. The remapping provides a better visualization of the stretching spatial distribution, but is not necessary for the global stretching time computation (3.19). For this toy model, the folding effects are dominant, and the inverse folding time well captures the spatial structure of $\alpha / t$. Yet, the inverse folding time diverges outside of the vortex where the streamline curvature tends to zero. Indeed, according to (3.11) zero curvature implies zero local frequency $f$, and thus infinite folding time [see (3.13)]. For such a weak curvature, the relevant model is the uniform shear. Following the space partition (3.13), the stretching time is chosen as a shearing time in these areas. The global time evolution models for the averaged stretching rate (3.19) and for the tracer gradients (4.17) are also successfully tested in Fig. 4.

The spectral roll-off proxy (4.9) is illustrated for the toy flow in Fig. 5. The local Gaussian approximation successfully captures the spectral tail shift toward small scales. The associated spatial fields have been presented in Fig. 1.

We also exemplify the adaptive filtering of the advected tracer [with squared correlation length (4.10)] in Fig. 6. The tracer is advected during a time $t$, and then smoothed by a Gaussian filter with the width (4.10). Hence, small-scale tracer structures are created by the advection and are then filtered out. However, the transport of large-scale tracer structures due to advection remains after filtering. The combined effect of advection and filtering moves the large-scale structures, but keeps the global amount of small-scale structures stationary.

\section{b. Ocean applications}

A similar analysis is performed using satellite data. Following geostrophic assumption, velocities are estimated from altimeter-derived SSH fields. We employ precomputed gridded geostrophic velocities from AVISO. The altimeter products were produced by SSALTO/Developing Use of
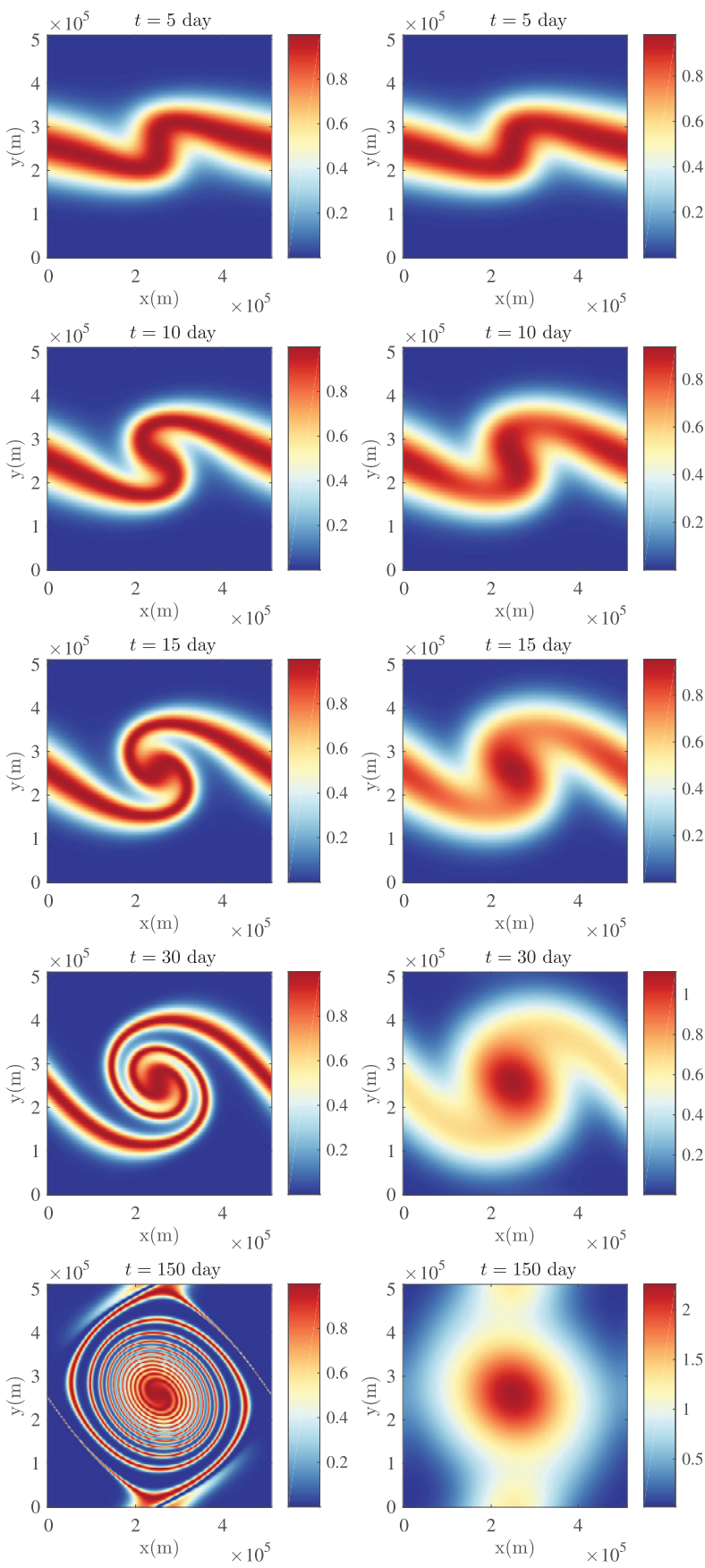

FIG. 6. (left) Tracer advected and (right) tracer advected and then smoothed by our adapted Gaussian filter for the toy model at (from top to bottom) $t=5,10,15,30$, and 150 days.

Altimetry for Climate Studies (DUACS) and distributed by AVISO, with support from CNES (http://www.aviso. altimetry.fr/duacs/). The velocity field, on which the Eulerian diagnostics will be estimated, corresponds to 1 January 2011, in the Antarctic Circumpolar Current (ACC) region, south of Australia. Nevertheless, for the reference Lagrangian advection, we will rely on (slowly) time-varying daily AVISO 

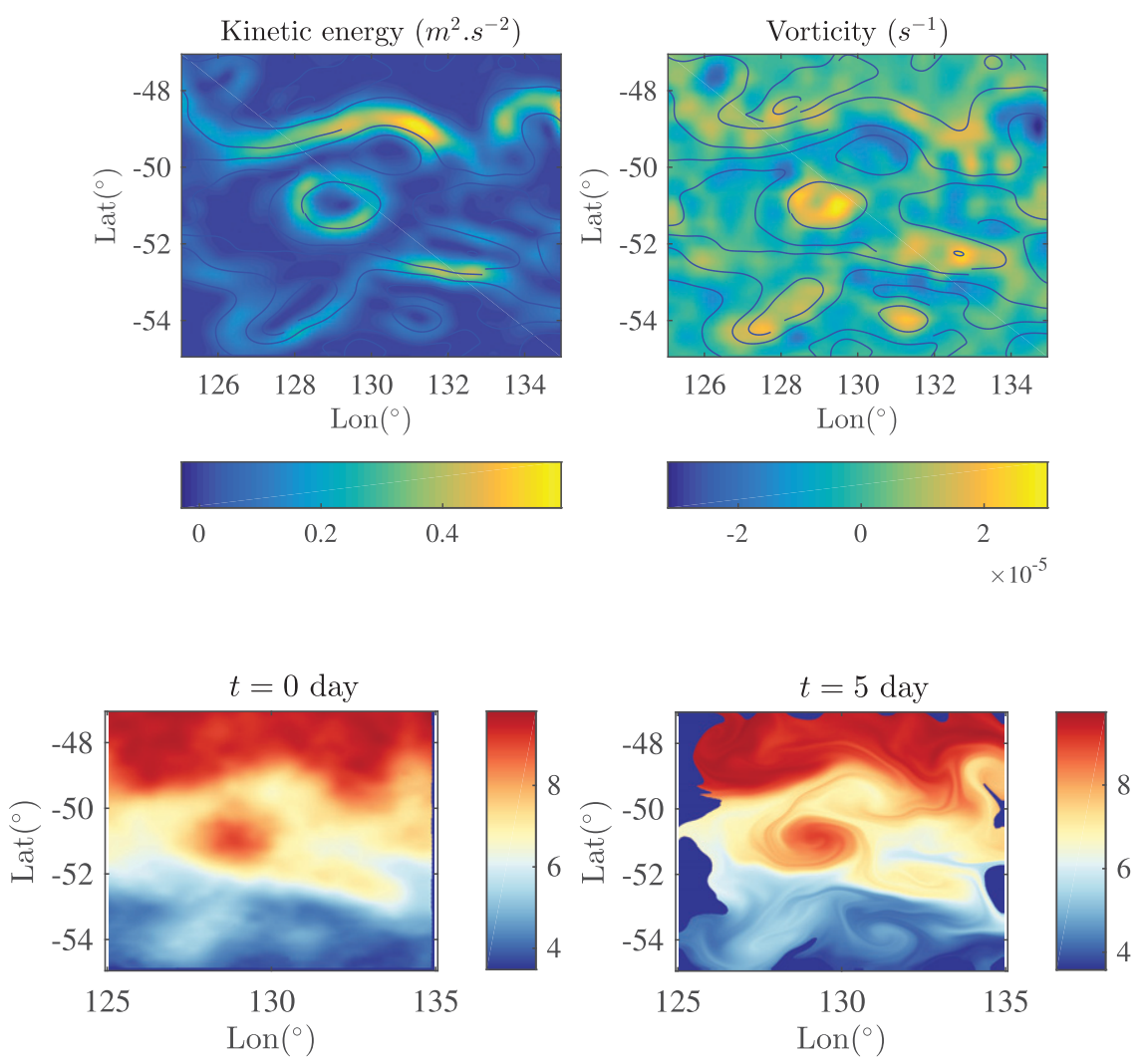

FIG. 7. (top left) Kinetic energy $\left(\mathrm{KE} ; \mathrm{m}^{2} \mathrm{~s}^{2}\right)$, (top right) vorticity $\left(\mathrm{s}^{-1}\right)$, (bottom left) SST $\left({ }^{\circ} \mathrm{C}\right)$, all measured by satellite the 1 Jan 2011, and (bottom right) SST $\left({ }^{\circ} \mathrm{C}\right)$ after a 5-day advection. In the top images, streamlines are superimposed. The streamlines, the KE, the vorticity, and the advection are defined by SSH-derived velocity fields.

dataset. A small spatial window of $10^{\circ} \times 10^{\circ}$ is first considered. For sea surface temperature, the Ifremer/ODYSSEA SST Level 4 product is considered. Produced daily using optimal interpolation (OI) on a global $0.1^{\circ}$ grid, it provides a daily cloud-free field of foundation sea surface temperature at approximately $10-\mathrm{km}$ resolution $\left(0.1^{\circ}\right)$ over the full globe. It is generated by merging microwave and infrared satellite observations.

Figure 7 delineates the kinetic energy (KE) and the vorticity fields. The KE shows the ACC eastward jet between latitudes $-50^{\circ}$ and $-48^{\circ}$ for 1 January 2011. Two (warm) anticyclones and a (cold) depression are visible both in the vorticity and initial SST fields at $\left(-51^{\circ}, 129^{\circ}\right),\left(-54^{\circ}, 131^{\circ}\right)$, and $\left(-49.5^{\circ}, 130.5^{\circ}\right)$, respectively. The 1 January 2011 , SST field is then advected. Similarly to Dencausse et al. (2014), time interpolation is performed to obtain the velocity fields between two daily data. The dipole closed to the jet creates a mushroom-like structure in the advected tracer. Each vortex wraps the tracer, creating spirals. The small southern anticyclone $\left(-54^{\circ}, 131^{\circ}\right)$ seems weaker than the other anticyclone $\left(-51^{\circ}, 129^{\circ}\right)$. Yet, it faster wraps the tracer, as velocities are certainly larger than over the dipole area.

Figure 8 shows the time-normalized growth rate $(\alpha / t)^{2}$, the mesochronic vorticity $\widetilde{\omega}$, and the weighting of the tracer/flow correlation $\beta / \alpha$. The squared inverse of folding, shearing and stretching times are also presented. A slight low-pass spatial filtering (2-km filter width) is applied to the stretching time to help distinguish the filamentary structures. The spatial distributions of time-normalized stretching rate and inverse squared stretching time are found very similar. The amplitude of the stretching time is slightly underestimated (ratio of about 2). In the aforementioned vortex boundaries, intense mixing occurs, whereas the inverse shearing time is weak. Comparable to the toy model results, folding effect due to differential rotations near the vortex boundaries is the leading mixing processes.

A larger ocean extend is now considered to encompass a broader variety of structures and dynamical processes. The spatial location and the date remain the same. Figure 9 displays the KE and the vorticity. The jet and many eddies are visible. The SST is again advected (Fig. 10). The advection creates small-scale structures, becoming nonphysical spirals when the advection time is too long. After 48 days, the advected domain is strongly deformed, especially by the eastward jet. Figure 11 compares the time-normalized stretching ratio, $(\alpha / t)^{2}$, and the estimated inverse squared stretching time in this larger spatial window. As found, most stretching structures are well predicted by the proposed model. 

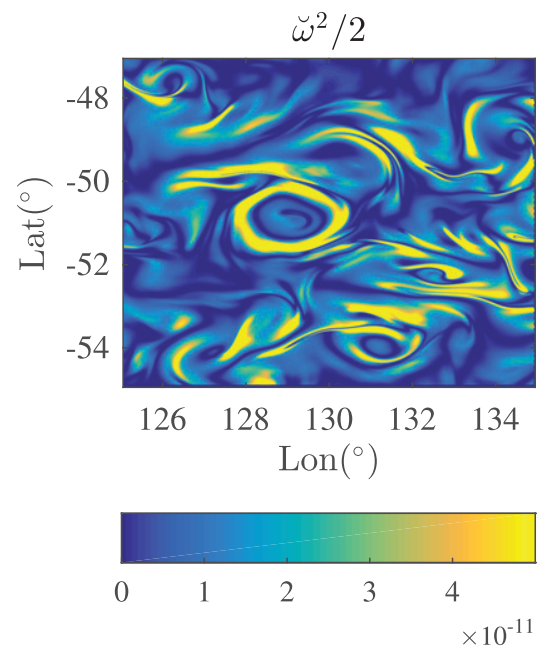

$\times 10^{-11}$
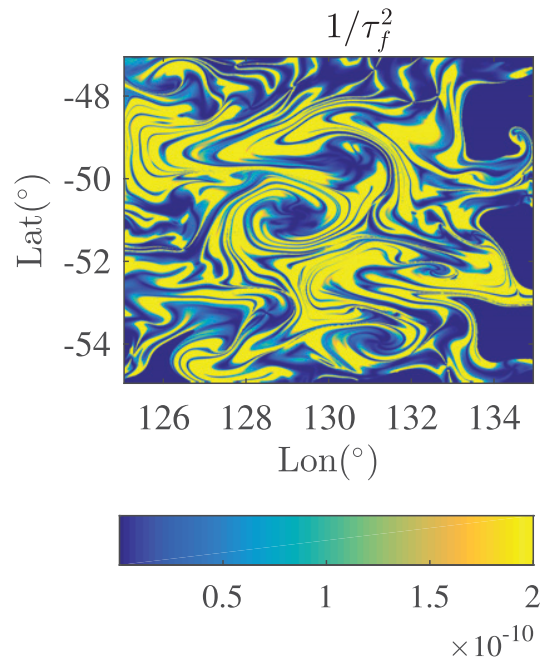
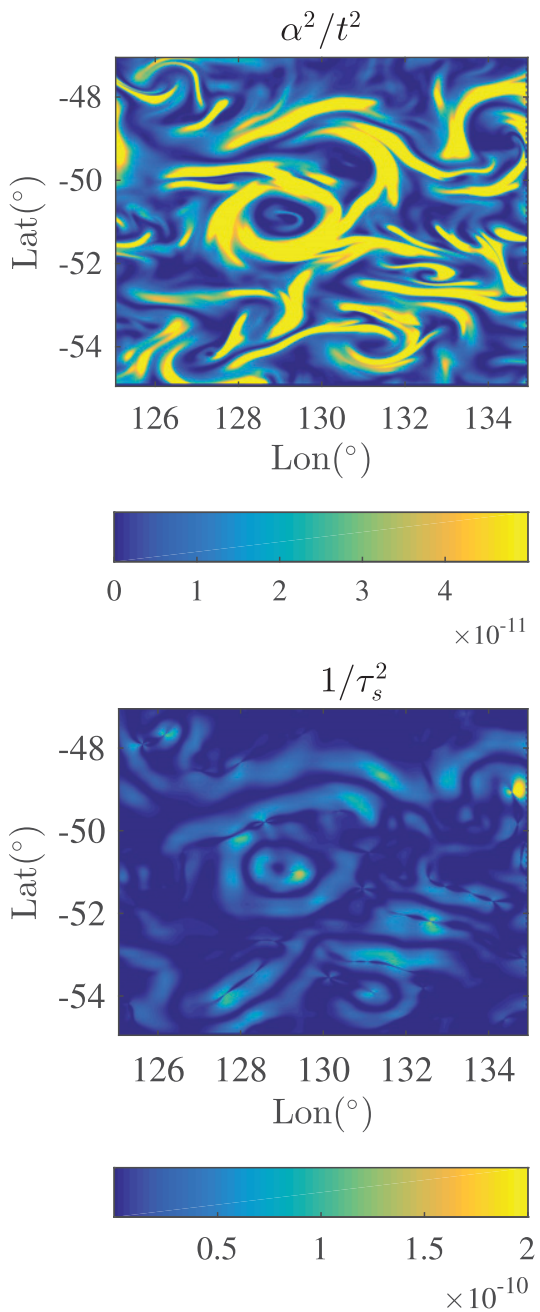
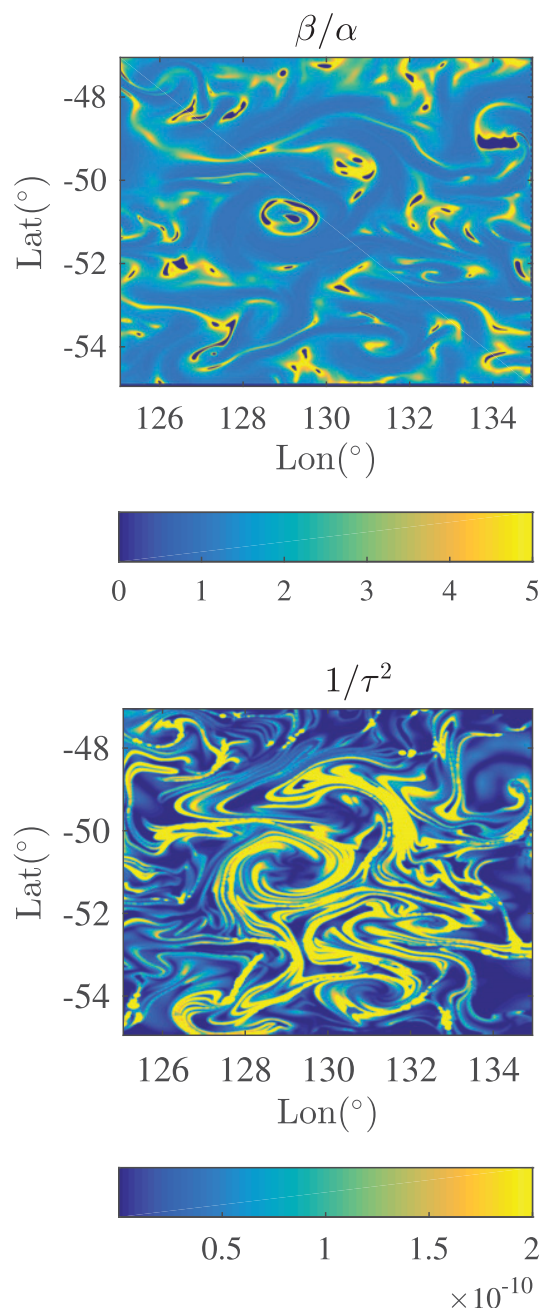

FIG. 8. Values of (top left) the mesochronic vorticity $\omega^{2} / 2\left(\mathrm{~s}^{-2}\right)$, (top center) the time-normalized stretching growth rate $(\alpha / t)^{2}\left(\mathrm{~s}^{-2}\right)$, and (top right) the ratio $\beta / \alpha$ (dimensionless) and (bottom left) the squared inverse of the folding time $\left(\mathrm{s}^{-2}\right)$, (bottom center) the shearing time $\left(\mathrm{s}^{-2}\right)$, and (bottom right) the stretching time $\left(\mathrm{s}^{-2}\right)$, in the initial grid (points $\mathbf{x}_{0}$ ) at time $t=5$ days for the SSH-derived velocity fields. We can observe the good match between the stretching rate and our Eulerian estimation of the inverse stretching time.

Finally, Fig. 12 presents the time evolution of the averaged stretching rate (3.19) and of the averaged tracer gradients norm (4.17). The reference plots clearly exhibit the structures prescribed by the derived models:

$$
\overline{\alpha^{2}}=\left(\frac{t}{\tau_{G}}\right)^{2} \text { and } \frac{\overline{\|\nabla T\|^{2}}}{\overline{\left\|\nabla T_{0}\right\|^{2}}}=\left\{\begin{array}{cl}
1 & \text { if } t \ll \tau_{G} \\
\left(\frac{t}{\tau_{G}}\right)^{2} & \text { if } t \gg \tau_{G}
\end{array} .\right.
$$

A global stretching time is estimated to 1.67 days. The plots reveal a good match, though the stretching time seems slightly underestimated (by a factor of about $\sim 1.7$ ). The small shift between predicted and reference-averaged tracer gradients norm may also be explained by a residual correlation between the tracer and the underlying flow as explained previously with Eq. (2.3).

Figure 13 illustrates the self-similarity-based estimate (4.16), applied to the ACC SST field. As prescribed, the spectrum tail slope of the advected SST reaches the value -3 after 5 days of advection, -2.5 after 10 days, and -2 after 48 days. The $-3,-2.5$, or -2 spectrum slope observed in "highresolution" tracer data can be physically relevant. For instance, a surface quasigeostrophic dynamics (Held et al. 1995 ) would lead to a $-5 / 3$ slope for the SST spectrum (if the salinity contribution to buoyancy is neglected). However, here $-3,-2.5$, and -2 are arbitrarily chosen values. Theoretically, any spectrum slope ( $<-1$ and $>$ to the initial slope) could be reached by this downscaling process. Figure 10 displays the spatial SST fields before and after advection. These data correspond to the summer season, January in the Southern Hemisphere.

A seasonal variation can then be studied. Figure 14 displays, for each day of the year 2011, the global stretching time, $\tau_{G}$, the spectrum slope of the measured SST and the prescribed advection time to reach a -2.5 spectrum slope. As 

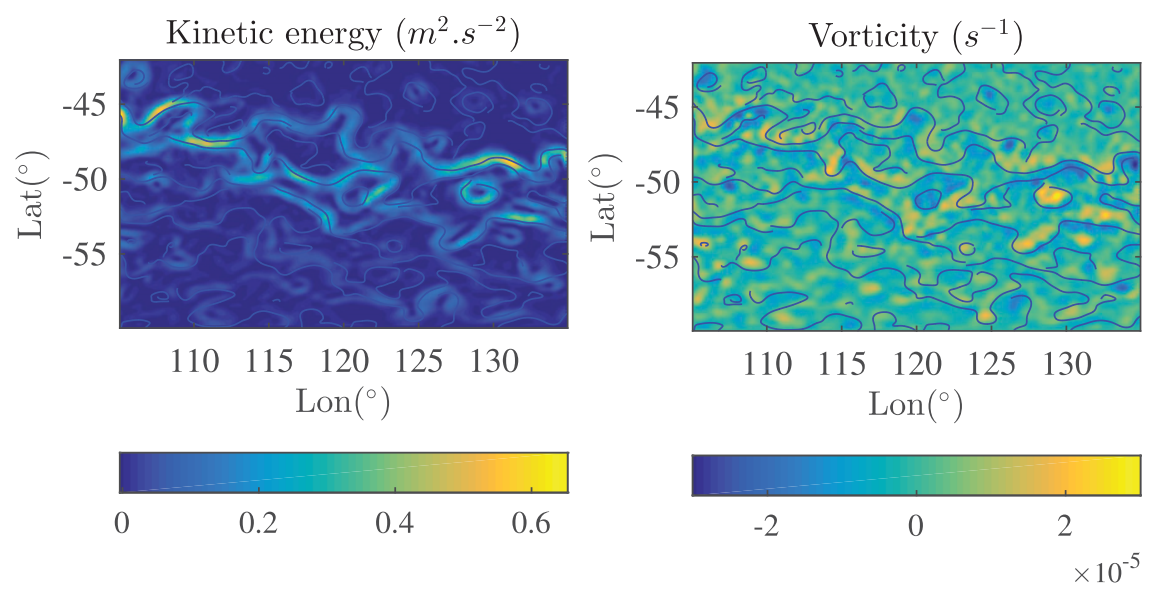

FIG. 9. (left) KE $\left(\mathrm{m}^{2} \mathrm{~s}^{2}\right)$ and (right) vorticity $\left(\mathrm{s}^{-1}\right)$ derived from SSH measured on 1 Jan 2011.

found, the stretching is faster during the winter. The measured SST spectral slopes are relatively stationary (close to -4$)$. Accordingly, the prescribed advection time is smaller in wintertime.

Berti and Lapeyre (2014) proposed other Eulerian estimates to prescribe the advection time: the inverse of the vorticity root-mean-square (RMS) $\left[\overline{\left(\nabla^{\perp} \cdot \mathbf{v}\right)^{2}}\right]^{-1 / 2}$ and of the velocity gradient $\mathrm{RMS}\left(\overline{\|\nabla \mathbf{v}\|^{2}}\right)^{-1 / 2}$. The latter is directly linked to the shearing time (3.7). Yet, these estimates can encode shearing but not folding. Indeed, folding involves second-order derivatives of the velocity, such as to describe the curvature variation of adjacent streamlines (3.13). Moreover, these criteria do not depend on the initial or on the resulting spectral slope. So, these criteria cannot fully control the necessary advection time, and may not be sufficient to control the resulting tracer spectral slopes. For instance, Fig. 14 demonstrates that these criteria strongly underestimate the advection time needed to reach a -2.5 spectrum slope. Accordingly, these criteria mostly apply to very short advection time, with a resulting advected tracer already close to the true SST.

\section{Conclusions}

In this paper, we analyzed how fluid parcels are stretched and folded by a smoothed velocity field, creating strong tracer gradients and raising the high-wavenumber part of the tracer spectral distribution. That is the case for SSH-inferred surface currents and the associated advection of coarse-scale tracer observations. Lagrangian methods, such as the determination
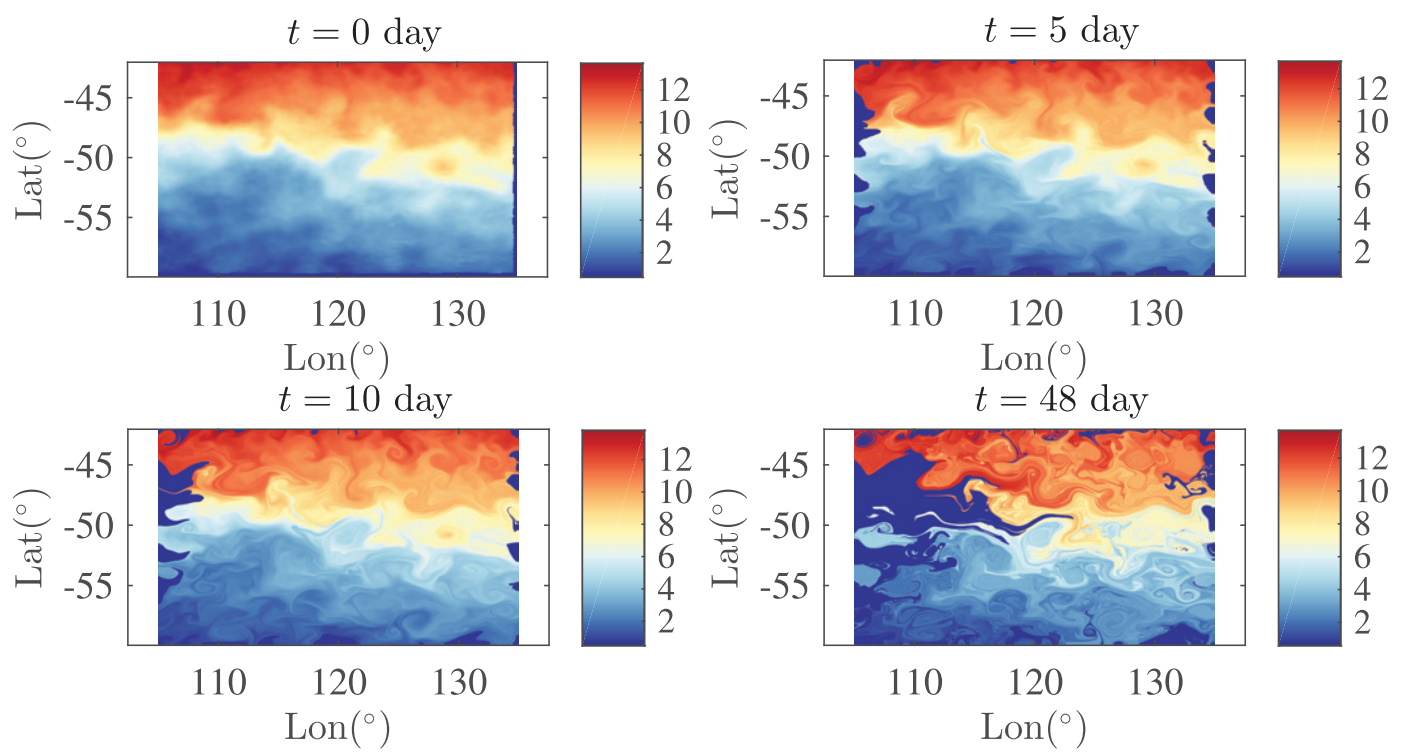

FIG. 10. (top left) SST $\left({ }^{\circ} \mathrm{C}\right)$ measured by satellite on 1 Jan 2011 after (top right) 5-, (bottom left) 10-, and (bottom right) 48-day advection. 

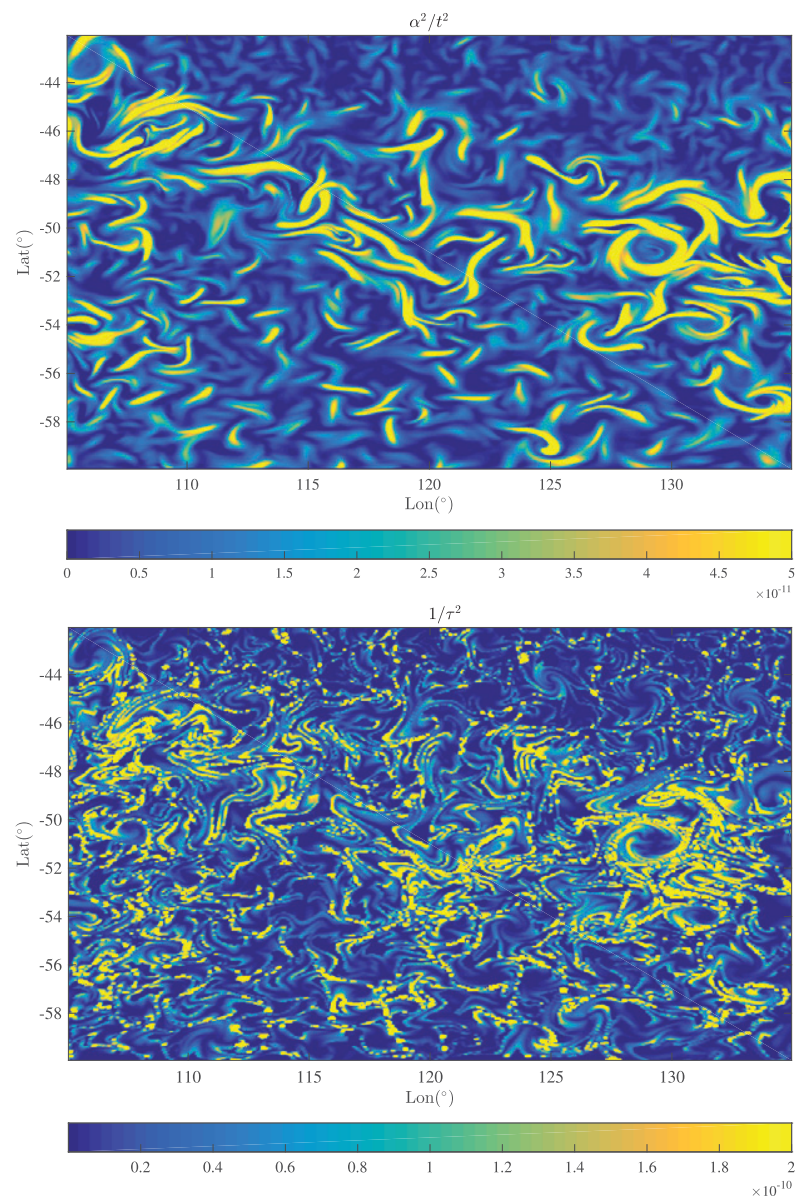

FIG. 11. (top) The time-normalized stretching growth rate $(\alpha / t)^{2}\left(\mathrm{~s}^{-2}\right)$ and (bottom) the squared inverse of the stretching time $1 / \tau^{2}\left(\mathrm{~s}^{-2}\right)$ in the initial grid (points $\mathbf{x}_{0}$ ) at time $t=5$ days.

of finite-time Lyapunov exponent (FTLE), are now very popular means to infer upper-ocean transport properties of heat, salt, nutrients or pollutants from such velocity fields. Still, Lagrangian methods can be computationally expensive to

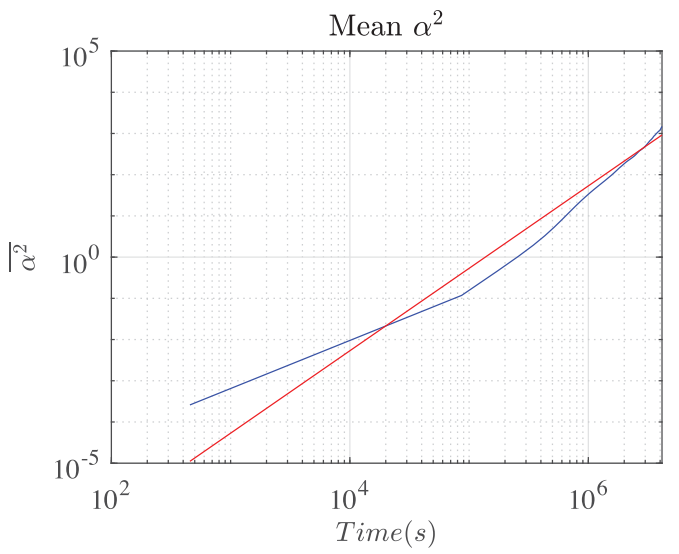

perform integration of particle along trajectories, and may not be suitable to quickly assess short-term material transport. Using Eulerian quantities from a single snapshot of velocities, our proposed development is more practical and fully exploit the connection between the Cauchy-Green deformation tensor and the evolution of the averaged squared norm of the advected tracer gradients.

Recently, following a variational theory to objectively define Eulerian coherent structures, Serra and Haller (2016) and Nolan et al. (2020) exploit the fact that for infinitesimally small integration times, the eigenvectors of the right Cauchy-Green strain tensor are equal to those of the Eulerian rate-ofstrain tensor. This property can thus already provide an Eulerian diagnostic, i.e., the instantaneous Lyapunov exponent structure, to help identify major flow features dominating short-time particle deformation patterns.

The present developments are not limited to small integration times. Two characteristics of the flow influence the norm of the advected tracer gradients: a local growth rate, associated with the eigenvalues of the Cauchy-Green tensor, and the orientation of the stable direction, eigenvector of the Cauchy-Green tensor. Integrated over space, the influence of local orientation disappears if the initial tracer is not correlated to the flow. This decorrelation can be due to a strong spatial smoothing induced by initial tracer observation process. It is then demonstrated that when the initial correlations between smooth divergent-free flow fields and tracers are weak the overall gradients can only strengthen. The local growth rate of the tracer gradients is independent of the initial tracer distribution, and is directly related to FTLEs and along-trajectory time-integrated velocities, i.e., mesochronic velocities.

In the case of SSH-inferred surface currents, Eulerian velocity fields are almost stationary during one or two weeks. As such, a simple and efficient prognosis can be derived. It extends the widely used Okubo-Weiss method-also known as the Q-criterion - which instantaneously compares relative vorticity to strain properties. Computationally cheap and perfectly suited to Eulerian snapshots of satellite-derived largescale ocean flows, our proposed criterion identifies regions where mixing can occur and quantifies it. The Eulerian

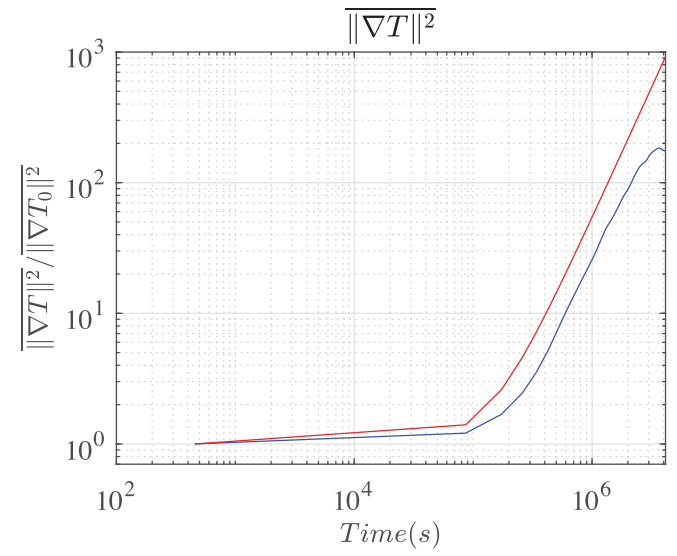

FIG. 12. (left) The averaged stretching rate $\overline{\alpha^{2}}$ and (right) the ratio of squared-norm mean of tracer gradients $\overline{\|\nabla T\|^{2}} /\left\|\nabla T_{0}\right\|^{2}$ for the satellite data, both in log-log plot along time. The blue line is the real value and the red line our model. 

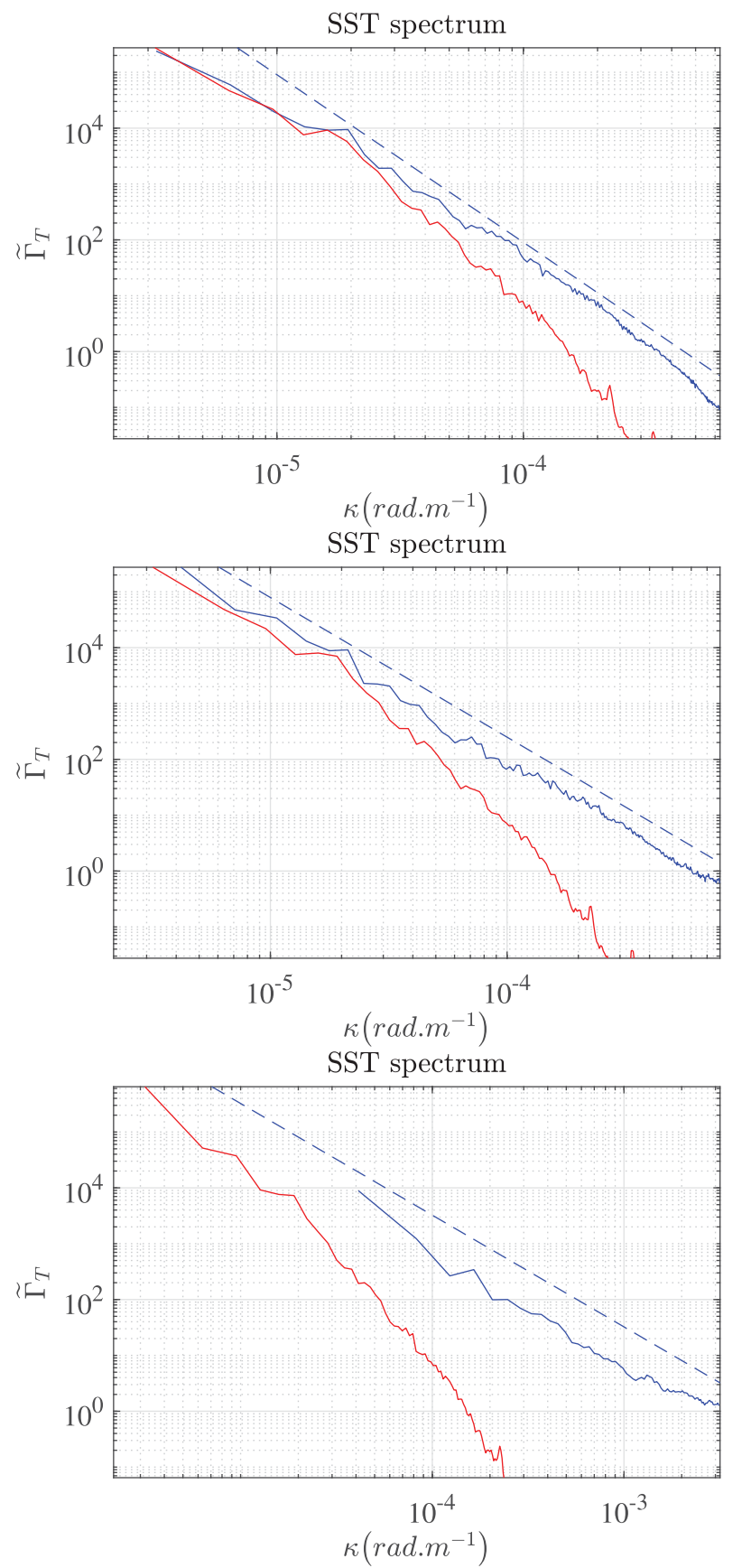

FIG. 13. SST spectrum of the satellite data (red) and after prescribed advection (blue) with (top) 5 days for a -3 spectrum slope, (middle) 10 days for a -2.5 spectrum slope, and (bottom) 48 days for a -2 spectrum slope. The expected spectrum slopes are superimposed (blue dashed line). The associated spatial fields are presented in Fig. 10. On the blue bottom spectrum, missing large-scale values are due to a strong deformation of the advected spatial domain by the jet (see Fig. 10).

descriptors can well separate mesoelliptic regions, rotating areas over which the tracer gradient norm is conserved, from mesohyperbolic regions where motion is dominated by stretching in
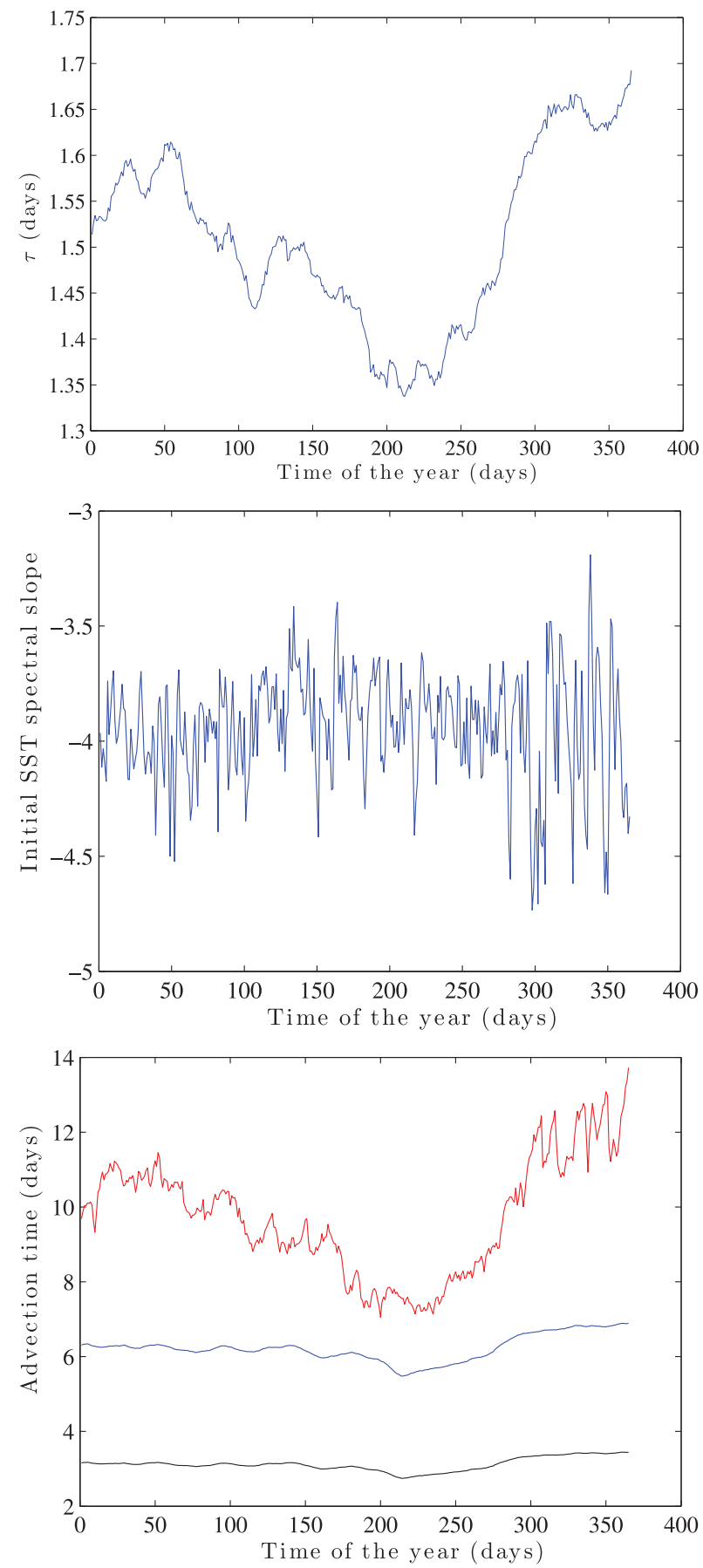

FIG. 14. (top) Global stretching time $\tau_{G}$ (in days), (middle) SST spectrum slope of Globcurrent data, and (bottom) prescribed advection time to reach a -2.5 spectrum slope (in days) with the velocity gradient RMS (blue line), vorticity RMS (black line), and our model (red line).

one direction and contraction in the other. Over these latter areas, strain and mesochronic vorticity compete and tracer gradients tend to increase. Moreover, our Eulerian proxies quantify the tracer gradients growth. 
Tracer gradients also control and specify the high-wavenumber tracer spectra. As presented and discussed, our prognostic analysis helps consistently determining the advection time and low-pass filter to apply when using Lagrangian downscaling advection methods. Based on these developments, a practical estimation of the horizontal diffusivity is also derived to help constraining subgrid parameterizations of large-scale flow simulations. The smooth velocity component acting to raise the high-wavenumber part of the spectrum (4.9), the horizontal effective diffusivity will balance this rise to best take into account unresolved small-scale components. Further investigations could thus extend the proposed criterion to stochastic flows, possibly exhibiting preferred sense of rotation, leading to quasi-stationary drift terms superposed to rapidly time-uncorrelated terms. The stochastic Eulerian framework proposed in Bauer et al. (2020), Mémin (2014), Resseguier et al. (2017a,b, 2020) shall be suited for such a purpose. Indeed, the slow-fast decomposition of the velocity can lead to introduce a smooth drift component, adding to the mesochronic velocity, and a random highly oscillating velocity component, acting to consistently define the diffusivity. The introduction of so-called polarized small-scale fluctuations (Middleton and Loder 1989) might then be tested to provide meaningful information on the additional folding/shearing effects associated to local statistical drifts attached to spatially distributed small-scale fluctuations (Bauer et al. 2020).

To further note, the Eulerian prognosis descriptors are certainly well suited to present-day large-scale altimeter-derived velocity estimates. Satellite-derived maps are weekly available, and time sequences of Eulerian estimates of flow mixing can be performed. Accordingly, seasonal to interannual variations of time evolution of folding and shearing properties of upper-ocean flows in different basins can also be derived. The simple prognostic shall then serve to possibly assess readjustments of the surface mesoscale ocean circulation over the last three decades (Martínez-Moreno et al. 2021).

Finally, the presented analytical and numerical results have strongly highlighted the importance of folding induced by upper-ocean eddies in tracers' dynamics. The actual satellite altimeter constellation will soon include a future wide-swath Surface Water and Ocean Topography (SWOT) altimeter (Morrow et al. 2019), to more precisely characterize ocean sea surface height variability. Specifically, ocean spatial structures will thus be better resolved. New Eulerian prognosis descriptors will thus be derived from these snapshot flow fields, and the impact of spatial resolution better assessed when compared to standard actual altimeter-derived products.

Acknowledgments. The authors acknowledge the support of the ESA DUE GlobCurrent and WOC projects, the "Laboratoires d'Excellence" CominLabs, Lebesgue and Mer through the SEACS project and the ERC EU project 856408-STUOD. The authors also acknowledge Guillaume Dencausse, Guillaume Lapeyre and Aurelien Ponte for helpful discussions. The authors deeply thank the reviewers and the associate editor. By their comments, suggestions, and questions they greatly helped us to improve our initial manuscript.
Data availability statement. These data were produced by ESA/GlobCurrent project and obtained from Ifremer/CERSAT. They are free and open.

\section{APPENDIX A}

\section{Recap on Cauchy-Green Tensor Analysis}

The Cauchy-Green tensor diagonalization writes as follows:

$$
\nabla \boldsymbol{\phi}^{\mathrm{T}}\left(\nabla \boldsymbol{\phi}^{\mathrm{T}}\right)^{\mathrm{T}}=\mathbf{P D P}^{\mathrm{T}} \text {, with } D_{i i}=1+\alpha^{2}\left[1-(-1)^{i} \frac{\beta}{\alpha}\right] \text {, }
$$

where $\mathbf{P}$ is an orthogonal matrix, $\alpha^{2}=(1 / 2)\left\|\nabla \boldsymbol{\phi}^{\mathrm{T}}\right\|^{2}-1 \geq 0$ and $\beta^{2}=\alpha^{2}+2$, using the Frobenius matrix norm. The eigenvalues $D_{i i}$ define the finite-time Lyapunov exponents (FTLE) (Haller and Yuan 2000; Thiffeault and Boozer 2001; Haller 2005; Haller and Sapsis 2011). Note that Pierrehumbert and Yang (1993) introduced this notion but provided an erroneous way of calculating it (referring to the frame-dependent flow map gradient eigenvalues instead of its frame-independent singular values). The largest and the smallest FTLEs are

$$
\Lambda=\frac{1}{2 t} \log \left(D_{11}\right) \text { and }-\Lambda=\frac{1}{2 t} \log \left(D_{22}\right) .
$$

The Cauchy-Green tensor encodes insightful and frameindependent information of the flow, further controlling the tracer gradient norm evolution (Haller and Yuan 2000).

\section{APPENDIX B}

\section{Exact Formula for the Tracer Gradient Norm}

Note that at a given time, the transported tracer gradient $\nabla T$ can be written from the initial gradient field $\nabla T_{0}$ as

$$
\nabla T(\mathbf{x})=\nabla\left\{T_{0}\left[\boldsymbol{\phi}^{-1}(\mathbf{x})\right]\right\}=\left(\nabla \boldsymbol{\phi}^{\mathrm{T}}\right)^{-1}\left[\boldsymbol{\phi}^{-1}(\mathbf{x})\right] \nabla T_{0}\left[\boldsymbol{\phi}^{-1}(\mathbf{x})\right] .
$$

Using the divergence-free assumption in the variable change and the matrix diagonalization (B1) with the identities $D_{11}^{-1}=D_{22}$ and $D_{22}^{-1}=D_{11}$, an exact expression of the averaged squared norm of tracer gradients reads

$$
\overline{\|\nabla T\|^{2}}-\overline{\left\|\nabla T_{0}\right\|^{2}}=\frac{1}{S} \int_{\Omega} d \mathbf{x}\|\nabla T(\mathbf{x})\|^{2}-\frac{1}{S} \int_{\Omega} d \mathbf{x}_{0}\left\|\nabla T_{0}\left(\mathbf{x}_{0}\right)\right\|^{2},
$$

$$
=\frac{1}{S} \int_{\Omega} d \mathbf{x}_{0}\left\|\left[\nabla \boldsymbol{\phi}^{\mathrm{T}}\right]^{-1}\left(\mathbf{x}_{0}\right) \nabla T_{0}\left(\mathbf{x}_{0}\right)\right\|^{2}-\frac{1}{S} \int_{\Omega} d \mathbf{x}_{0}\left\|\nabla T_{0}\left(\mathbf{x}_{0}\right)\right\|^{2},
$$

$$
=\overline{\left(\nabla T_{0}\right)^{\mathrm{T}}\left\{\left[\nabla \boldsymbol{\phi}^{\mathrm{T}}\left(\nabla \boldsymbol{\phi}^{\mathrm{T}}\right)^{\mathrm{T}}\right]^{-1}-\mathbb{I}_{d}\right\} \nabla T_{0},}
$$




$$
=\alpha^{2}(\underbrace{\left(1-\frac{\beta}{\alpha}\right)}_{<0}\left(\mathbf{P}^{\mathrm{T}} \nabla T_{0}\right)_{1}^{2}+\underbrace{\left(1+\frac{\beta}{\alpha}\right)}_{>0}\left(\mathbf{P}^{\mathrm{T}} \nabla T_{0}\right)_{2}^{2}),
$$

where $\left(\mathbf{P}^{\mathrm{T}} \nabla T_{0}\right)_{i}$ is the $i$ th component of the vector $\mathbf{P}^{\mathrm{T}} \nabla T_{0}$. Thus, the Cauchy-Green tensor and the initial tracer gradient completely determine the averaged squared norm of advected tracer gradients. To simplify the above expression, we define the angle between the tracer gradient and the compressive (stable) direction of the direct flow as

$$
\cos \left(\theta_{T_{0}}^{\boldsymbol{\phi}}\right)=\frac{\left(\mathbf{P}^{\mathrm{T}} \nabla T_{0}\right)_{2}}{\left\|\nabla T_{0}\right\|} .
$$

Finally, we infer the following compact expression:

$$
\overline{\|\nabla T\|^{2}}-\overline{\left\|\nabla T_{0}\right\|^{2}}=\overline{\left\|\nabla T_{0}\right\|^{2} \alpha^{2}\left[1+\frac{\beta}{\alpha} \cos \left(2 \theta_{T_{0}}^{\phi}\right)\right]} .
$$

\section{APPENDIX C}

Mixing Criterion of Mezić et al. (2010)

As derived in Mezić et al. (2010), the incompressibility of the flow yields

$$
1=\operatorname{det}\left(\nabla \boldsymbol{\phi}^{\mathrm{T}}\right)=\operatorname{det}\left(\mathbb{I}_{d}+t \nabla \breve{\mathbf{v}}^{\mathrm{T}}\right)=1+t \operatorname{tr}\left(\nabla \nabla^{\mathrm{T}}\right)+t^{2} \operatorname{det}\left(\nabla \breve{\mathbf{v}}^{\mathrm{T}}\right) .
$$

For an incompressible flow, the mesochronic velocity follows

$$
t \operatorname{det}\left(\nabla \breve{\mathbf{v}}^{\mathrm{T}}\right)=-\operatorname{tr}\left(\nabla \breve{\mathbf{v}}^{\mathrm{T}}\right)=-\nabla \cdot \breve{\mathbf{v}} \neq 0 .
$$

The definition of the local growth rate $\alpha^{2}$ then reads

$$
\begin{aligned}
\alpha^{2} & \triangleq \frac{1}{2}\left\|\nabla \boldsymbol{\phi}^{\mathrm{T}}\right\|^{2}-1, \\
& =\frac{1}{2}\left\|\mathbb{I}_{d}+t \nabla \breve{\mathbf{v}}^{\mathrm{T}}\right\|^{2}-1, \\
& =-t\left[t \operatorname{det}\left(\nabla \overline{\mathbf{v}}^{\mathrm{T}}\right)\right]+\frac{t^{2}}{2}\left\|\nabla \overline{\mathbf{v}}^{\mathrm{T}}\right\|^{2}, \\
& =\frac{t^{2}}{2}\left[\left(\partial_{x} \breve{u}-\partial_{y} \breve{v}\right)^{2}+\left(\partial_{y} \breve{u}+\partial_{x} \breve{v}\right)^{2}\right] .
\end{aligned}
$$

Then, the incompressibility constraint (C2) helps rewrite (C6) as a function of the determinant $\operatorname{det}\left(\nabla_{\mathbf{v}}^{\mathrm{T}}\right)$ :

$$
\begin{aligned}
\alpha^{2} & =\frac{t^{2}}{2}\left[(\nabla \cdot \breve{\mathbf{v}})^{2}-4 \operatorname{det}\left(\nabla \breve{\mathbf{v}}^{\mathrm{T}}\right)+\breve{\omega}^{2}\right], \\
& =\frac{t^{2}}{2}\left\{t^{2} \operatorname{det}\left(\nabla \breve{\mathbf{v}}^{\mathrm{T}}\right)\left[\operatorname{det}\left(\nabla \breve{\mathbf{v}}^{\mathrm{T}}\right)-\frac{4}{t^{2}}\right]+\breve{\omega}^{2}\right\} .
\end{aligned}
$$

\section{APPENDIX D}

\section{Folding Time}

Denote the derivatives of $\mathbf{g}$ as follows:

$\left(\partial_{1} \mathbf{g}^{\mathrm{T}}\right)\left(\mathbf{z}_{1}, z_{2}\right)=\nabla_{\mathbf{z}_{1}}\left[\mathbf{g}^{\mathrm{T}}\left(\mathbf{z}_{1}, z_{2}\right)\right]$ and $\left(\partial_{2} \mathbf{g}\right)\left(\mathbf{z}_{1}, z_{2}\right)=\partial_{z_{2}}\left[\mathbf{g}\left(\mathbf{z}_{1}, z_{2}\right)\right]$.

with both terms 1 - periodic with respect to its second variable. Using frequency invariance (3.10), we can replace $f\left(\mathbf{x}_{0}\right)$ by $f\left[\boldsymbol{\phi}\left(\mathbf{x}_{0}, t\right)\right]$ in the model (3.8):

$$
\boldsymbol{\phi}\left(\mathbf{x}_{0}, t\right)=\mathbf{x}_{0}+\mathbf{g}\left\{\mathbf{x}_{0}, f\left[\boldsymbol{\phi}\left(\mathbf{x}_{0}, t\right)\right] t\right\} .
$$

Then, replacing back $f\left[\boldsymbol{\phi}\left(\mathbf{x}_{0}, t\right)\right]$ by $f\left(\mathbf{x}_{0}\right)$ after evaluating the gradient, the stretching of the flow reads

$$
\begin{array}{r}
\nabla \boldsymbol{\phi}^{\mathrm{T}}\left(\mathbf{x}_{0}, t\right)=\mathbb{I}_{d}+\left(\partial_{1} \mathbf{g}^{\mathrm{T}}\right)\left\{\mathbf{x}_{0}, f\left[\boldsymbol{\phi}\left(\mathbf{x}_{0}, t\right)\right] t\right\} \\
+t \nabla \boldsymbol{\phi}^{\mathrm{T}}\left(\mathbf{x}_{0}, t\right) \nabla f\left[\boldsymbol{\phi}\left(\mathbf{x}_{0}, t\right)\right]\left(\partial_{2} \mathbf{g}^{\mathrm{T}}\right)\left\{\mathbf{x}_{0}, f\left[\boldsymbol{\phi}\left(\mathbf{x}_{0}, t\right)\right] t\right\},
\end{array}
$$

$$
\begin{aligned}
& =\mathbb{I}_{d}+\left(\partial_{1} \mathbf{g}^{\mathrm{T}}\right)\left[\mathbf{x}_{0}, f\left(\mathbf{x}_{0}\right) t\right] \\
& +t \nabla \phi^{\mathrm{T}}\left(\mathbf{x}_{0}, t\right) \nabla f\left[\phi\left(\mathbf{x}_{0}, t\right)\right]\left(\partial_{2} \mathbf{g}^{\mathrm{T}}\right)\left[\mathbf{x}_{0}, f\left(\mathbf{x}_{0}\right) t\right] .
\end{aligned}
$$

In the last equality, the second right-hand term is time periodic and thus bounded. If we neglect its time variation (e.g., for large advection time $t$ ), it writes

$$
\left(\partial_{1} \mathbf{g}^{\mathrm{T}}\right)\left[\mathbf{x}_{0}, f\left(\mathbf{x}_{0}\right) t\right] \approx\left(\partial_{1} \mathbf{g}^{\mathrm{T}}\right)\left(\mathbf{x}_{0}, 0\right)=\nabla \boldsymbol{\phi}^{\mathrm{T}}\left(\mathbf{x}_{0}, 0\right)-\mathbb{I}_{d}=0 .
$$

Introducing the original periodic model (3.8) into its definition, the Lagrangian velocity $\mathbf{V}$ reads

$$
\mathbf{V}\left(\mathbf{x}_{0}, t\right)=\frac{d \boldsymbol{\phi}\left(\mathbf{x}_{0}, t\right)}{d t}=f\left(\mathbf{x}_{0}\right)\left(\partial_{2} \mathbf{g}\right)\left[\mathbf{x}_{0}, f\left(\mathbf{x}_{0}\right) t\right] .
$$

Finally, the flow gradient expression (D4) can be rewritten using Eqs. (D5) and (D6):

$$
\begin{aligned}
\nabla \boldsymbol{\phi}^{\mathrm{T}}\left(\mathbf{x}_{0}, t\right) & =\mathbb{I}_{d}+t \nabla \boldsymbol{\phi}^{\mathrm{T}}\left(\mathbf{x}_{0}, t\right) \frac{\nabla f\left[\boldsymbol{\phi}\left(\mathbf{x}_{0}, t\right)\right]}{f\left(\mathbf{x}_{0}\right)} \mathbf{V}^{\mathrm{T}}\left(\mathbf{x}_{0}, t\right), \\
& =\mathbb{I}_{d}+t \nabla \boldsymbol{\phi}^{\mathrm{T}}\left(\mathbf{x}_{0}, t\right)\left(\frac{1}{f} \nabla f \mathbf{v}^{\mathrm{T}}\right)\left[\boldsymbol{\phi}\left(\mathbf{x}_{0}, t\right)\right],
\end{aligned}
$$

where the frequency invariance (3.10) was used in the last equality. Factorizing terms in $\nabla \boldsymbol{\phi}^{\mathrm{T}}$,

$$
\mathbb{I}_{d}=\nabla \boldsymbol{\phi}^{\mathrm{T}}\left(\mathbf{x}_{0}, t\right)\left\{\mathbb{I}_{d}-t\left(\frac{1}{f} \nabla f \mathbf{v}^{\mathrm{T}}\right)\left[\boldsymbol{\phi}\left(\mathbf{x}_{0}, t\right)\right]\right\},
$$

we then inverse the matrix equation and remap with the inverse flow $\boldsymbol{\phi}^{-1}$ :

$$
\left(\nabla \boldsymbol{\phi}^{\mathrm{T}}\right)^{-1}\left[\boldsymbol{\phi}^{-1}(\mathbf{x}, t), t\right]=\mathbb{I}_{d}-t\left(\frac{1}{f} \nabla f \mathbf{v}^{\mathrm{T}}\right)(\mathbf{x}) .
$$


Since the frequency is a function of the streamline (3.10), we have

$$
0=\frac{d}{d t}\left[f\left(\mathbf{x}_{0}\right)\right]=\frac{d}{d t}\left\{f\left[\boldsymbol{\phi}\left(\mathbf{x}_{0}, t\right)\right]\right\}=(\mathbf{v} \cdot \nabla f)\left[\boldsymbol{\phi}\left(\mathbf{x}_{0}, t\right), t\right]
$$

Therefore, the frequency gradient is orthogonal to the velocity, and

$$
\|\nabla f\| \approx\left|\frac{\mathbf{v}^{\perp}}{\left\|\mathbf{v}^{\perp}\right\|} \cdot \nabla f\right| .
$$

Eigenvalues of the Cauchy-Green tensor are the inverse of one another. Therefore, their traces are equal, and hence the matrix $\nabla \boldsymbol{\phi}^{\mathrm{T}}$ and its inverse have the same Frobenius norm (equals to that trace). So, the time dependency of the growth rate in the final grid (points $\mathbf{x}$ ) follows from its definition and from (D10):

$$
\begin{aligned}
\alpha^{2}\left[\boldsymbol{\phi}^{-1}(\mathbf{x}, t), t\right] & \triangleq \frac{1}{2}\left\|\nabla \boldsymbol{\phi}^{\mathrm{T}}\left[\boldsymbol{\phi}^{-1}(\mathbf{x}, t), t\right]\right\|^{2}-1, \\
& =\frac{1}{2}\left\|\left(\nabla \boldsymbol{\phi}^{\mathrm{T}}\right)^{-1}\left[\boldsymbol{\phi}^{-1}(\mathbf{x}, t), t\right]\right\|^{2}-1, \\
& =-\underbrace{\left(\frac{t}{f} \nabla f \cdot \mathbf{v}\right)}_{=0 \text { by }(\mathrm{D} 11)}(\mathbf{x})+\left(\frac{t^{2}}{2 f^{2}}\|\nabla f\|^{2}\left\|\mathbf{v}^{2}\right\|\right)(\mathbf{x}) .
\end{aligned}
$$

\section{APPENDIX E}

\section{Spectra of Tracers for Smooth Flow}

With Dirichlet boundary conditions for the tracer, integration by parts leads to

$$
\begin{aligned}
-\mathbf{H}_{\gamma_{q}}(0) & =-\left[\nabla_{\delta} \mathbf{x} \nabla_{\delta} \mathbf{x}^{\mathrm{T}} \gamma_{q}(\boldsymbol{\delta} \mathbf{x})\right]_{\delta_{\delta_{x}=0}}, \\
& =-\frac{1}{S} \int_{\Omega} d \mathbf{x} q(\mathbf{x})\left[\nabla_{\delta} \mathbf{x} \nabla_{\delta} \mathbf{x}^{\mathrm{T}} q(\mathbf{x}+\boldsymbol{\delta} \mathbf{x})\right]_{\left.\right|_{\delta_{x}=0}}, \\
& =-\frac{1}{S} \int_{\Omega} d \mathbf{x} q(\mathbf{x}) \mathbf{H}_{q}(\mathbf{x}) \\
& =\frac{1}{S} \int_{\Omega} d \mathbf{x} \nabla q(\mathbf{x})[\nabla q(\mathbf{x})]^{\mathrm{T}} \\
& =\frac{\nabla q(\nabla q)^{\mathrm{T}}>0(\text { in the Lowner sense) }}{\nabla}
\end{aligned}
$$

In particular, the Hessian trace simplified to

$$
\operatorname{tr}\left[\mathbf{H}_{\gamma_{q}}(0)\right]=-\overline{\operatorname{tr}\left[\nabla q(\nabla q)^{\mathrm{T}}\right]}=-\overline{\|\nabla q\|^{2}}
$$

The Fourier transform of the covariance expression (4.3) provides the approximation of the spectrum tail:

$$
\begin{gathered}
\Gamma_{q}(\mathbf{k}) \triangleq|\hat{q}(\mathbf{k})|^{2}=\hat{\gamma}_{q}(\mathbf{k}) \\
\underset{\|\mathbf{k}\| \rightarrow \infty}{\sim} \frac{2 \pi\left(\overline{q^{2}}\right)^{2}}{\operatorname{det}\left[\mathbf{H}_{\gamma_{q}}(0)\right]^{1 / 2}} \times \exp \left\{-\frac{1}{2} \mathbf{k}^{\mathrm{T}}\left[-\overline{q^{2}} \mathbf{H}_{\gamma_{q}}^{-1}(0)\right] \mathbf{k}\right\},
\end{gathered}
$$

where the hat denotes spatial Fourier transform. Nevertheless, $\nabla q(\nabla q)^{\mathrm{T}}$ and thus $\mathbf{H}_{\gamma_{q}}$ is not convenient to manipulate. Accordingly, hereafter, we will focus on the omnidirectional spectrum of a scalar $q$. This will enable us to replace $\nabla q(\nabla q)^{\mathrm{T}}$ by $\|\nabla q\|$ in the expression of the spectrum (E7).

$\Gamma_{q}(\kappa)=\kappa \oint_{[0,2 \pi]} d \theta_{\mathbf{k}} \Gamma_{f}(\mathbf{k})$,

$=\kappa \oint_{[0,2 \pi]} d \theta_{\mathbf{k}} \int_{\Omega} d \boldsymbol{\delta} \mathbf{x} \gamma_{q}(\boldsymbol{\delta} \mathbf{x}) e^{-i \mathbf{k} \cdot \boldsymbol{\delta}} \mathbf{x}$

$$
\begin{gathered}
=\kappa \oint_{[0,2 \pi]} d \boldsymbol{\delta} \mathbf{x}\left\{\gamma_{q}(0)-\frac{1}{2} \boldsymbol{\delta} \mathbf{x}^{\mathrm{T}} \frac{1}{S} \int_{\Omega} d \mathbf{x} \nabla q(\mathbf{x})[\nabla q(\mathbf{x})]^{\mathrm{T}} \boldsymbol{\delta} \mathbf{x}\right. \\
\left.+\underset{\|\boldsymbol{\delta} \mathbf{x}\| \rightarrow 0}{o}\left(\|\boldsymbol{\delta} \mathbf{x}\|^{3}\right)\right\} \times e^{-i \mathbf{k} \cdot \boldsymbol{\delta} \mathbf{x}}, \\
=\frac{\kappa}{S} \oint_{[0,2 \pi]} d \theta_{\mathbf{k}} \int_{\Omega} d \boldsymbol{\delta} \mathbf{x} \int_{\Omega} d \mathbf{x}\left\{q^{2}(\mathbf{x})-\frac{1}{2} \boldsymbol{\delta} \mathbf{x}^{\mathrm{T}} \nabla q(\mathbf{x})[\nabla q(\mathbf{x})]^{\mathrm{T}} \boldsymbol{\delta} \mathbf{x}\right. \\
\left.+\underset{\|\boldsymbol{\delta} \mathbf{x}\| \rightarrow 0}{o}\left(\left\|\boldsymbol{\delta} \mathbf{x}^{3}\right\|\right)\right\} \times e^{-i \mathbf{k} \cdot \boldsymbol{\delta} \mathbf{x}}
\end{gathered}
$$

$$
\begin{gathered}
=\frac{\kappa}{S} \int_{\Omega} d \mathbf{x} \oint_{[0,2 \pi]} d \theta_{\mathbf{k}} \int_{\Omega} d \boldsymbol{\delta} \mathbf{x}\left\{q^{2}(\mathbf{x})-\frac{1}{2}\left[\frac{\nabla q(\mathbf{x})}{\|\nabla q(\mathbf{x})\|} \cdot \delta \mathbf{x}\right]^{2}\|\nabla q(\mathbf{x})\|^{2}\right. \\
\left.+\underset{\|\boldsymbol{\delta} \mathbf{x}\| \rightarrow 0}{o}\left(\|\boldsymbol{\delta} \mathbf{x}\|^{3}\right)\right\} e^{-i \mathbf{k} \cdot \boldsymbol{\delta} \mathbf{x}} .
\end{gathered}
$$

Locally in $\mathbf{x}$, we can define a variable change for $\boldsymbol{\delta} \mathbf{x}=\left(\delta x_{1}\right.$ $\left.\delta x_{2}\right)^{\mathrm{T}}$. We apply the rotation matrix $\mathbf{U}(\mathbf{x})=[1 /\|\nabla q(\mathbf{x})\|]$ $\left[\nabla q(\mathbf{x}) \nabla^{\perp} q(\mathbf{x})\right]$ to $\delta \mathbf{x}$ to align $\delta \mathbf{x}$ with the tracer gradient and denote $\theta(\mathbf{x})$ the angle of the associated rotation:

$$
\begin{aligned}
\Gamma_{q}(\kappa)= & \frac{\kappa}{S} \int_{\Omega} d \mathbf{x} \oint_{[0,2 \pi]} d \theta_{\mathbf{k}} \int_{\Omega} d \boldsymbol{\delta} \mathbf{x}\left[q^{2}(\mathbf{x})-\frac{1}{2}\left(\delta \mathbf{x}_{1}\right)^{2}\|\nabla q(\mathbf{x})\|^{2}\right. \\
& \left.+\underset{\|\boldsymbol{\delta} \mathbf{x}\| \rightarrow 0}{O}\left(\|\boldsymbol{\delta} \mathbf{x}\|^{3}\right)\right] \times e^{-i\left(\mathbf{U}^{\mathrm{T}} \mathbf{k}\right) \cdot \boldsymbol{\delta} \mathbf{x}} \\
= & \frac{\kappa}{S} \int_{\Omega} d \mathbf{x} \oint_{[\theta(\mathbf{x}), \theta(\mathbf{x})+2 \pi]} d \theta_{\mathbf{k}} \int_{\Omega} d \boldsymbol{\delta} \mathbf{x}\left[q^{2}(\mathbf{x})-\frac{1}{2}\left(\delta x_{1}\right)^{2} \nabla q(\mathbf{x})_{2}^{2}\right. \\
& \left.+\underset{\|\mathbf{\delta} \mathbf{x}\| \rightarrow 0}{O}\left(\|\boldsymbol{\delta} \mathbf{x}\|^{3}\right)\right] \times e^{-i \mathbf{k} \cdot \boldsymbol{\delta} \mathbf{x}}
\end{aligned}
$$




$$
\begin{aligned}
= & \frac{\kappa}{S} \int_{\Omega} d \mathbf{x} \oint_{[0,2 \pi]} d \theta_{\mathbf{k}} \int_{\Omega} d \boldsymbol{\delta} \mathbf{x}\left[q^{2}(\mathbf{x})-\frac{1}{2}\left(\delta x_{1}\right)^{2}\|\nabla q(\mathbf{x})\|^{2}\right. \\
& \left.+\underset{\|\delta \mathbf{x}\| \rightarrow 0}{o}\left(\|\boldsymbol{\delta} \mathbf{x}\|^{3}\right)\right] \times e^{-i \mathbf{k} \cdot \boldsymbol{\delta} \mathbf{x}}, \\
= & \kappa \oint_{[0,2 \pi]} d \theta_{\mathbf{k}} \int_{\Omega} d \boldsymbol{\delta} \mathbf{x}\left[\overline{q^{2}}-\frac{1}{2}\left(\delta x_{1}\right)^{2} \overline{\|\nabla q\|^{2}}\right. \\
& \left.+\underset{\|\delta \mathbf{x}\| \rightarrow 0}{o}\left(\|\boldsymbol{\delta} \mathbf{x}\|^{3}\right)\right] e^{-i \mathbf{k} \cdot \boldsymbol{\delta} \mathbf{x}} .
\end{aligned}
$$

The third equality above is due to the averaging over the spatial frequency angle $\theta_{\mathbf{k}}$. Indeed, $\mathbf{U}^{\mathrm{T}} \mathbf{k}$ is just a rotation of k. And, integrating over $[0,2 \pi]$ or over $[\theta(\mathbf{x}), 2 \pi+\theta(\mathbf{x})]$ is the same thing, since it leads to the same closed line: a circle of radius $\kappa$.

$$
\begin{aligned}
& \Gamma_{q}(\kappa)= \kappa \oint_{[0,2 \pi]} d \theta_{\mathbf{k}} \int_{\Omega} d \boldsymbol{\delta} \mathbf{x}_{1} d \boldsymbol{\delta} \mathbf{x}_{2}\left\{\overline{q^{2}} \exp \left[-\frac{1}{2} \frac{\overline{\|\nabla q\|^{2}}}{\overline{q^{2}}}\left(\delta x_{1}\right)^{2}\right]\right. \\
&\left.+\underset{\|\boldsymbol{\delta} \mathbf{x}\| \rightarrow 0}{o}\left(\|\boldsymbol{\delta} \mathbf{x}\|^{3}\right)\right\} \times e^{-i k_{1} \delta x_{1}} e^{-i k_{2} \delta x_{2}} \\
& \underset{\|\mathbf{k}\| \rightarrow \infty}{\sim} \oint_{[0,2 \pi]} \kappa d \theta_{\mathbf{k}} \frac{C_{f}}{2(2 \pi)} \exp \left(-\frac{1}{2} L_{f}^{2} k_{1}^{2}\right)(2 \pi) \delta\left(k_{2}\right)
\end{aligned}
$$

where $\mathbf{k}=\left(k_{1} k_{2}\right)^{\mathrm{T}}=[\kappa \cos (\theta k) \kappa \sin (\theta k)]^{\mathrm{T}}, L_{f}^{2}=\overline{q^{2}} / \overline{\|\nabla q\|^{2}}$ and $C_{f} /[2(2 \pi)]=\left[2 \pi\left(\overline{q^{2}}\right)^{3} / \overline{\|\nabla q\|^{2}}\right]^{1 / 2}$. Note that the asymptotic equivalence is an approximation. Then, switching from cylindrical to Cartesian coordinates in each half ring $\left\{\mathbf{k} \in \mathbb{R}^{2} \mid k_{1} \leq 0,\|\mathbf{k}\|=\kappa\right\}$ and $\left\{\mathbf{k} \in \mathbb{R}^{2} \mid k_{1} \geq 0,\|\mathbf{k}\|=\kappa\right\}$ yields

$$
\tilde{\Gamma}_{q}(\kappa) \underset{\|\mathbf{k}\| \rightarrow \infty}{\sim} 2 \int_{-\kappa}^{\kappa} \frac{d k_{2}}{\sqrt{1-\left(\frac{k_{2}}{\kappa}\right)^{2}}} \frac{C_{f}}{2} \exp \left[-\frac{1}{2} L_{f}^{2}\left(\kappa^{2}-k_{2}^{2}\right)\right] \delta\left(k_{2}\right)
$$

$$
\underset{\|\mathbf{k}\| \rightarrow \infty}{\sim} C_{f} \exp \left(-\frac{1}{2} L_{f}^{2} \kappa^{2}\right) .
$$

\section{REFERENCES}

Aref, H., 1984: Stirring by chaotic advection. J. Fluid Mech., 143, 1-21, https://doi.org/10.1017/S0022112084001233.

Batchelor, G. K., 1959: Small-scale variation of convected quantities like temperature in turbulent fluid Part 1. General discussion and the case of small conductivity. J. Fluid Mech., 5, 113-133, https://doi.org/10.1017/S002211205900009X.

Bauer, W., P. Chandramouli, B. Chapron, L. Li, and E. Mémin, 2020: Deciphering the role of small-scale inhomogeneity on geophysical flow structuration: A stochastic approach. $J$.
Phys. Oceanogr., 50, 983-1003, https://doi.org/10.1175/JPO-D19-0164.1.

Berti, S., and G. Lapeyre, 2014: Lagrangian reconstructions of temperature and velocity in a model of surface ocean turbulence. Ocean Modell., 76, 59-71, https://doi.org/10.1016/j. ocemod.2014.02.003.

Dencausse, G., R. Morrow, M. Rogé, and S. Fleury, 2014: Lateral stirring of large-scale tracer fields by altimetry. Ocean Dyn., 64, 61-78, https://doi.org/10.1007/s10236-013-0671-8.

d'Ovidio, F., J. Isern-Fontanet, C. López, E. Hernández-García, and E. García-Ladona, 2009: Comparison between Eulerian diagnostics and finite-size Lyapunov exponents computed from altimetry in the Algerian basin. Deep-Sea Res. I, 56, 1531, https://doi.org/10.1016/j.dsr.2008.07.014.

Dufau, C., M. Orsztynowicz, G. Dibarboure, R. Morrow, and P.-Y. Le Traon, 2016: Mesoscale resolution capability of altimetry: Present and future. J. Geophys. Res. Oceans, 121, 4910-4927, https://doi.org/10.1002/2015JC010904.

Falkovich, G., K. Gawçdzki, and M. Vergassola, 2001: Particles and fields in fluid turbulence. Rev. Mod. Phys., 73, 913-975, https://doi.org/10.1103/RevModPhys.73.913.

Gower, J., K. Denman, and R. Holyer, 1980: Phytoplankton patchiness indicates the fluctuation spectrum of mesoscale oceanic structure. Nature, 288, 157-159, https://doi.org/10. 1038/288157a0.

Hadjighasem, A., M. Farazmand, D. Blazevski, G. Froyland, and G. Haller, 2017: A critical comparison of Lagrangian methods for coherent structure detection. Chaos, 27, 053104, https:// doi.org/10.1063/1.4982720.

Haller, G., 2005: An objective definition of a vortex. J. Fluid Mech., 525, 1-26, https://doi.org/10.1017/S0022112004002526.

— 2016: Dynamic rotation and stretch tensors from a dynamic polar decomposition. J. Mech. Phys. Solids, 86, 70-93, https:// doi.org/10.1016/j.jmps.2015.10.002.

, and G. Yuan, 2000: Lagrangian coherent structures and mixing in two-dimensional turbulence. Physica D, 147, 352-370, https://doi.org/10.1016/S0167-2789(00)00142-1.

- and T. Sapsis, 2011: Lagrangian coherent structures and the smallest finite-time Lyapunov exponent. Chaos, 21, 023115, https://doi.org/10.1063/1.3579597.

— , A. Hadjighasem, M. Farazmand, and F. Huhn, 2016: Defining coherent vortices objectively from the vorticity. J. Fluid Mech., 795, 136-173, https://doi.org/10.1017/jfm.2016.151.

Held, I., R. Pierrehumbert, S. Garner, and K. Swanson, 1995: Surface quasi-geostrophic dynamics. J. Fluid Mech., 282, 1-20, https://doi.org/10.1017/S0022112095000012.

Karrasch, D., 2015: Attracting Lagrangian coherent structures on Riemannian manifolds. Chaos, 25, 087411, https://doi.org/10. 1063/1.4928451.

Klein, P., and Coauthors, 2019: Ocean-scale interactions from space. Earth Space Sci., 6, 795-817, https://doi.org/10.1029/ 2018EA000492.

Lehahn, Y., F. d'Ovidio, M. Lévy, and E. Heifetz, 2007: Stirring of the northeast Atlantic spring bloom: A Lagrangian analysis based on multisatellite data. J. Geophys. Res., 112, C08005, https://doi.org/10.1029/2006JC003927.

Lesieur, M., and R. Sardouny, 1981: Satellite-sensed turbulent ocean structure. Nature, 294, 673, https://doi.org/10.1038/ 294673a0.

Ma, T., N. Ouellette, and E. Bollt, 2016: Stretching and folding in finite time. Chaos, 26, 023112, https://doi.org/10.1063/1. 4941256. 
Martínez-Moreno, J., A. M. Hogg, M. H. England, N. C. Constantinou, A. E. Kiss, and A. K. Morrison, 2021: Global changes in oceanic mesoscale currents over the satellite altimetry record. Nat. Climate Change, 11, 397-403, https://doi.org/10. 1038/s41558-021-01006-9.

Mémin, E., 2014: Fluid flow dynamics under location uncertainty. Geophys. Astrophys. Fluid Dyn., 108, 119-146, https://doi.org/ 10.1080/03091929.2013.836190.

Mezić, I., S. Loire, V. Fonoberov, and P. Hogan, 2010: A new mixing diagnostic and gulf oil spill movement. Science, 330, 486-489, https://doi.org/10.1126/science.1194607.

Middleton, J. F., and J. W. Loder, 1989: Skew fluxes in polarized wave fields. J. Phys. Oceanogr., 19, 68-76, https://doi.org/10. 1175/1520-0485(1989)019<0068:SFIPWF $>2.0 . \mathrm{CO} ; 2$.

Morrow, R., and Coauthors, 2019: Global observations of finescale ocean surface topography with the Surface Water and Ocean Topography (SWOT) mission. Front. Mar. Sci., 6, 232, https://doi.org/10.3389/fmars.2019.00232.

Nolan, P. J., M. Serra, and S. D. Ross, 2020: Finite-time Lyapunov exponents in the instantaneous limit and material transport. Nonlinear Dyn., 100, 3825-3852, https://doi.org/10.1007/ s11071-020-05713-4.

Okubo, A., 1970: Horizontal dispersion of floatable particles in the vicinity of velocity singularities such as convergences. Deep-Sea Res. Oceanogr. Abstr., 17, 445-454, https://doi.org/ 10.1016/0011-7471(70)90059-8.

Pierrehumbert, R., and H. Yang, 1993: Global chaotic mixing on isentropic surfaces. J. Atmos. Sci., 50, 2462-2480, https://doi. org/10.1175/1520-0469(1993)050<2462:GCMOIS > 2.0.CO;2.

Price, J., M. Reed, M. Howard, W. Johnson, Z.-G. Ji, C. Marshall, N. Guinasso, and G. Rainey, 2006: Preliminary assessment of an oil-spill trajectory model using satellite-tracked, oil-spillsimulating drifters. Environ. Modell. Software, 21, 258-270, https://doi.org/10.1016/j.envsoft.2004.04.025.

Resseguier, V., E. Mémin, and B. Chapron, 2017a: Geophysical flows under location uncertainty, Part I: Random transport and general models. Geophys. Astrophys. Fluid. Dyn., 111, 149-176, https://doi.org/10.1080/03091929.2017.1310210.

- - and $\longrightarrow$, 2017b: Geophysical flows under location uncertainty, Part III: SQG and frontal dynamics under strong turbulence conditions. Geophys. Astrophys. Fluid. Dyn., 111, 209-227, https://doi.org/10.1080/03091929.2017.1312102.

— , W. Pan, and B. Fox-Kemper, 2020: Data-driven versus selfsimilar parameterizations for stochastic advection by lie transport and location uncertainty. Nonlinear Processes Geophys., 27, 209-234, https://doi.org/10.5194/npg-27-209-2020.

Rogé, M., R. A. Morrow, and G. Dencausse, 2015: Altimetric Lagrangian advection to reconstruct Pacific Ocean fine-scale surface tracer fields. Ocean Dyn., 65, 1249-1268, https://doi. org/10.1007/s10236-015-0872-4.

Serra, M., and G. Haller, 2016: Objective Eulerian coherent structures. Chaos, 26, 053110, https://doi.org/10.1063/1.4951720.

Shivamoggi, B., and G, van Heijst, 2011: The Okubo-Weiss criteria in two-dimensional hydrodynamic and magnetohydrodynamic flows. arXiv, 13 pp., https://arxiv.org/abs/1110.6190.

Thiffeault, J.-L., 2004: Stretching and curvature of material lines in chaotic flows. Physica D, 198, 169-181, https://doi.org/10. 1016/j.physd.2004.04.009.

— , and A. Boozer, 2001: Geometrical constraints on finite-time Lyapunov exponents in two and three dimensions. Chaos, 11, 16-28, https://doi.org/10.1063/1.1342079.

Vallis, G., 2006: Atmospheric and Oceanic Fluid Dynamics: Fundamentals and Large-Scale Circulation. Cambridge University Press, 769 pp.

Weiss, J., 1991: The dynamics of enstrophy transfer in two-dimensional hydrodynamics. Physica D, 48, 273-294, https://doi.org/ 10.1016/0167-2789(91)90088-Q.

Welander, P., 1955: Studies on the general development of motion in a two-dimensional, ideal fluid. Tellus, 7, 141-156, https:// doi.org/10.3402/tellusa.v7i2.8797. 